\title{
Tutorial on fiber-based sources for biophotonic applications
}

James R. Taylor 


\title{
Tutorial on fiber-based sources for biophotonic applications
}

\author{
James R. Taylor* \\ Imperial College, Femtosecond Optics Group, Physics Department, Prince Consort Road, London SW7 2BW, United Kingdom
}

\begin{abstract}
Fiber-based lasers and master oscillator power fiber amplifier configurations are described. These allow spectral versatility coupled with pulse width and pulse repetition rate selection in compact and efficient packages. This is enhanced through the use of nonlinear optical conversion in fibers and fiber-coupled nonlinear crystals, which can be integrated to provide all-fiber pump sources for diverse application. The advantages and disadvantages of sources based upon supercontinuum generation, stimulated Raman conversion, four-wave mixing, parametric generation and difference frequency generation, allowing spectral coverage from the UV to the mid-infrared, are considered. () The Authors. Published by SPIE under a Creative Commons Attribution 3.0 Unported License. Distribution or reproduction of this work in whole or in part requires full attribution of the original publication, including its DOI. [DOI: 10.1117/1. JBO.21.6.061010]
\end{abstract}

Keywords: nonlinear fiber optics; fiber lasers; ultrashort pulses; mode locked fiber lasers; solitons; fiber supercontinua; master oscillator power fiber amplifier; wavelength tunability.

Paper 160085SSVR received Feb. 11, 2016; accepted for publication May 19, 2016; published online Jun. $10,2016$.

\section{Introduction}

The vast range of applications and investigations in the broad and diverse biophotonics area is currently met by an equally vast range of conventional optical and laser sources. Most certainly, the practical demands of wavelength, pulse duration, repetition rate and average, as well as peak power levels cannot be met by one single source; however, an overall simplification of source technology will undoubtedly underpin a revolution in diagnostic techniques, particularly where the sources can be made compact, efficient, and effectively "hands free" in operation, in addition to a low cost of ownership. Some of these specific source requirements can be and are met by laser diode technology, but in very many instances, use must still be made of large-frame solid-state lasers in tandem with unwieldy solid-state regenerative amplifier schemes and bulk nonlinear optical configurations for additional wavelength diversity, which all occupy large footprints and complicate experimental configurations. Admittedly, these work and they have underpinned a vast catalogue of innovation in biophotonic technology; however, greater versatility and source simplification are particularly commercially attractive in addition to the unquestionable benefit in the research laboratory.

Over the past two decades, the fiber laser in its two dominant guises, the high average power laser and the ultrashort pulse light source, has made remarkable progress. Technological advances in fiber structures and manufacture coupled with equally remarkable progress in semiconductor pump laser technology and multimode optical fiber power combiners have underpinned the power scaling of the fiber laser, such that up to $10-\mathrm{kW}$ average power is commercially available from single-mode structures. ${ }^{1}$ With femtosecond pulses, peak powers in the megawatt range and average

*Address all correspondence to: James R. Taylor, E-mail: jr.taylor@ imperial.ac .uk powers in excess of $100 \mathrm{~W}$ can be achieved using optimized chirped pulse amplification configurations, again with $\mathrm{Yb}$ based systems. ${ }^{2}$ Although such peak and average powers are far beyond those required for biomedical application, the underlying base technology of the master oscillator power fiber amplifier (MOPFA) $)^{3}$ is an essential tool, particularly in adding spectral and temporal diversity, albeit at substantially lower power levels, for application in this area.

A schematic of the fully fiber-integrated MOPFA concept followed by a nonlinear conversion stage is shown in Fig. 1. The master oscillator seed source can take the form of mode locked laser (fiber, diode, or solid state) or be a directly modulated laser source, which allows greater versatility in parameter control. These seed parameters can vary from continuous wave (CW)to picosecond duration and at a selectable repetition rate. Integration with a high single-pass gain fiber amplifier leads to a robust, "hands-free" configuration.

To date, the greatest technical development and power scaling has been directed toward $\mathrm{Yb}$-doped silica-based fiber laser schemes with a gain spectrum approximately covering the range from 970 to $1100 \mathrm{~nm}$; however, the communications relevant Erbased systems operating from about 1520 to $1600 \mathrm{~nm}$ and Tmdoped silica fiber amplifiers operating from 1800 to $2000 \mathrm{~nm}$ provide additional coverage in the infrared. No other rare earth-doped silica fiber lasers are available in the near infrared and systems in the visible are not available either. Although bismuth-doped silica-based fiber lasers and amplifiers have been reported in the spectral range from 1150 to $1550 \mathrm{~nm}$ in differing glass compositions and impressive power scaling obtained, ${ }^{4}$ commercial systems are still not yet available. As a consequence, nonlinear optical conversion techniques have to be applied to obtain fiber-based systems with continuous wavelength operation throughout the visible and in the spectral voids between the established rare earth-based schemes. As some of these processes form the basis of the sources described herein, it is essential that a brief description of some of the contributing nonlinear effects is briefly presented. 


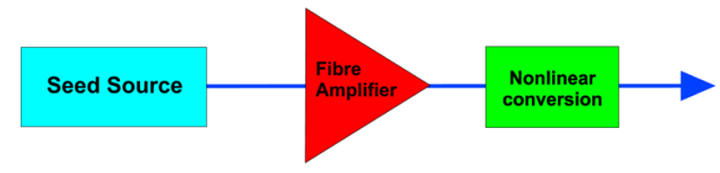

Fig. 1 A schematic of a fiber integrated master oscillator-power fiber amplifier (MOPFA) followed by nonlinear wavelength conversion.

\section{Nonlinear Optics in Fibers}

Nonlinear optics did not result from the discovery of the laser; for example, both Kerr and Pockels had investigated birefringence in transparent media as a result of an applied dc electric field. On the discovery of the laser by Maiman, however, the magnitude of the optical field itself was sufficient to observe nonlinear optical processes. With the introduction of the pulsed laser and, in particular, the techniques of Q-switching and mode locking with picosecond and femtosecond pulses being routinely and controllably generated with the latter, it became possible to generate power densities in excess of a terawatt per square centimeter in the focal region of a simple convex lens, with the associated electric field strength exceeding a megavolt per centimeter. As a consequence, any transparent medium placed in the focal region responded nonlinearly, and to describe the induced polarization, higher order terms of the electric field need to be considered, such that

$P=\varepsilon_{0}\left(\chi^{(1)} E+\chi^{(2)} E^{2}+\chi^{(3)} E^{3}+\ldots\right)$,

where $P$ is the polarization, $\varepsilon_{0}$ is the permittivity of free space and $\chi^{(n)}$ is the $n$ 'th order susceptibility. The first term on the right hand side represents the linear response under low field strength $E$. When driven by a field of frequency $\omega$, the second term gives rise to a response at frequency $2 \omega$, second harmonic generation, the first nonlinear effect to be observed using laser pumping. ${ }^{5}$ This term also results in sum and difference frequency mixing and optical rectification. In a medium that exhibits a center of symmetry, for example, silica fiber, this second order term vanishes to zero and although second harmonic generation has been observed in silica fibers, the conversion efficiencies obtained are unable to compete with those achieved in noncentrosymmetric crystals. In optical fiber, it is the third order term that contributes to the host of highly efficient nonlinear processes that are observed and that are briefly considered below.

In bulk optics, the interaction length of the focused beam is limited by the confocal parameter $\left(2 \pi w_{0}^{2} / \lambda\right)$, where $w_{0}$ is the beam waist and $\lambda$ is the wavelength of the light. For a beam waist of about $10 \mu \mathrm{m}$, the confocal parameter is $\sim 1 \mathrm{~mm}$. If, however, the pump radiation is focused into a single-mode fiber with a core diameter of around $10 \mu \mathrm{m}$, the interaction length in the fiber is simply limited by the loss. With fiber loss, clearly being dependent on wavelength but with optimized loss in modern fibers readily achieving $0.2 \mathrm{~dB} / \mathrm{km}$, loss lengths in excess of $10 \mathrm{~km}$ can be easily achieved. Consequently, singlemode fibers offer nonlinear interaction lengths over six orders of magnitude greater than can be achieved with bulk coupling, meaning that many of the nonlinear processes observed in bulk can be reproduced in fiber at substantially lower peak pump powers, while it must be remembered that silica also exhibits one of the lowest nonlinear coefficients. In addition, some of the processes require phase matching and, consequently, dispersion plays an equally important role in determining the efficiency of the generation process.

\subsection{Dispersion}

Although a linear effect, as discussed above, dispersion can play a vital role in the conversion efficiency of a nonlinear interaction in fiber. Dispersion arises as a result of the frequency dependence of the effective refractive index of a guided mode. Contributions arise from both the material and the waveguide structure. In addition, modal dispersion can have a very significant role in the phase matching of nonlinear processes, such as four-wave mixing (FWM); however, only single-mode operation is considered in this article. The group velocity $v_{\mathrm{g}}$ defines the propagation of a packet of monochromatic radiation (over an infinitesimal bandwidth) and is related to the propagation constant $\beta$ through the first derivative with respect to the frequency $\left(\beta_{1}=d \beta /\right.$ $d \omega=1 / v_{\mathrm{g}}$ ). The different group velocities of pulses at different wavelengths can set a limit on the nonlinear interaction length, which can be substantially less than the physical length of the optical fiber used, due to the associated pulse walkoff. Group velocity matching can be achieved by allowing the interacting pulses to straddle the dispersion zero wavelength. In conventional silicabased single-mode fiber, this is achievable at a minimum wavelength of $1270 \mathrm{~nm}$ and with dispersion-shifted fibers of conventional structure can be placed at wavelengths longer than this. The introduction of photonic crystal fiber, ${ }^{6,7}$ however, has had a remarkable impact on nonlinear fiber optics and through judicious design, the zero dispersion can be manipulated to be at any wavelength, even throughout the complete visible.

Due to the different group velocities of the spectral components of a pulse, temporal broadening (or temporal compression, depending on the initial chirp of the input pulse) can occur on propagation in an optical fiber. This group velocity dispersion is characterized by the second derivative of $\beta$. In practical units, the group delay dispersion $D$ is defined in units of $\mathrm{ps} /(\mathrm{nm} \mathrm{km})$ and defined by $D=-\left(2 \pi c / \lambda^{2}\right) \beta_{2}$, where $\beta_{2}$ is the group velocity dispersion, generally written in units of $\mathrm{s}^{2} \mathrm{~m}^{-1}$, where $\beta_{2}=d^{2} \beta / d \omega^{2}$. When $\beta_{2}$ is positive, the dispersion is normal, i.e., low frequency components travel with a greater velocity than high frequency components and when $\beta_{2}$ is negative, the dispersion is anomalous. To give an idea of the importance of dispersion, if one considers a transform limited pulse of 100 ps at $1.060 \mu \mathrm{m}$ (bandwidth $\sim 0.0165 \mathrm{~nm})$ in $100 \mathrm{~m}$ of standard single-mode telecommunications fiber $(D \sim 50 \mathrm{ps} /(\mathrm{nm} \mathrm{km})$, a relatively insignificant broadening of 0.0825 ps will take place, yet a transform-limited 1 -ps pulse will broaden by $8.25 \mathrm{ps}$, consequently reducing the peak intensity and reducing the nonlinear interaction. Correspondingly, a transform-limited 100-fs pulse would broaden to over $1 \mathrm{ps}$ in only $2 \mathrm{~m}$ of such a fiber. Even from the aspect of dispersion control, the impact of photonic crystal fibers (PCFs) on the field of nonlinear fiber optics is quite apparent. Through the selection of the pitch and diameter of the photonic crystal network around the core, the zero dispersion wavelength can be precisely controlled, such that the more commonly used pulsed laser excitation sources, such as Ti:Sapphire, $\mathrm{Nd}$ :YAG, or Yb-doped silica fiber, can operate in the region of low dispersion. Additionally, PCFs can be made to operate in a single transverse mode throughout the full bandwidth of transmission $^{8}$ or the mode field diameter can be significantly reduced giving rise to a substantially higher nonlinear 
parameter, ${ }^{9}$ leading to reduced required pump powers or shorter interaction lengths to achieve nonlinear conversion.

\subsection{Intensity-Dependent Refractive Index, Self-Phase Modulation and Optical Solitons}

The third order term in the equation for the induced polarization gives rise to both elastic and nonelastic nonlinear scattering processes. One of the most commonly encountered of the former is that of self-phase modulation (SPM) of a pulse, or cross-phase modulation when there is a simultaneous copropagating pulse. Because of the intensity-dependent refractive index, where $n=n_{0}+n_{2} I(t)$, the time-dependent intensity of a pulse gives rise to a corresponding modulation of the local refractive index. In silica, the time scale of the nonlinear response is of the order of a few femtoseconds and so can be treated as effectively instantaneous on the time scale of most commonly encountered incident pulsewidths. The induced modulation of the refractive index gives rise to a time-dependent phase shift, which gives a corresponding frequency shift that is simply the negative time derivative of the phase shift, as is shown in Fig. 2, while the instantaneous response time gives rise to symmetrical spectral broadening of the pulse on propagation.

As proposed by Shimizu, ${ }^{10}$ the process gives rise to the generation of new frequencies and a linear chirp over the central region of the pulse, with the frequency increasing in time-a positive chirp, as illustrated in Fig. 2. Alone, SPM has no effect on the temporal profile of the pulse; however, in association with dispersion, a temporal reshaping takes place. In optical fiber, SPM was first characterized by Ippen et al. ${ }^{11}$ On propagation in the region of normal dispersion, SPM will give rise to temporal broadening and a linearization of the chirp. As recognized by Treacy, ${ }^{12}$ the dispersive chirp can be compensated to second order by propagation through a grating pair, giving pulse compression and laying the foundation of the highly versatile fibergrating pulse compressor, ${ }^{13}$ allowing the production of pulses of a few tens of femtoseconds.

When SPM takes place in the anomalously dispersive region, where high-frequency components travel with a greater velocity than lower frequencies, then, with reference to Fig. 2, where SPM causes the high-frequency components to lie at the rear of the pulse, this will lead to pulse compression and power enhancement, which will lead to further spectral broadening. It can be envisaged that under certain circumstances, the linear effect of dispersion and the nonlinear effect of SPM could reach

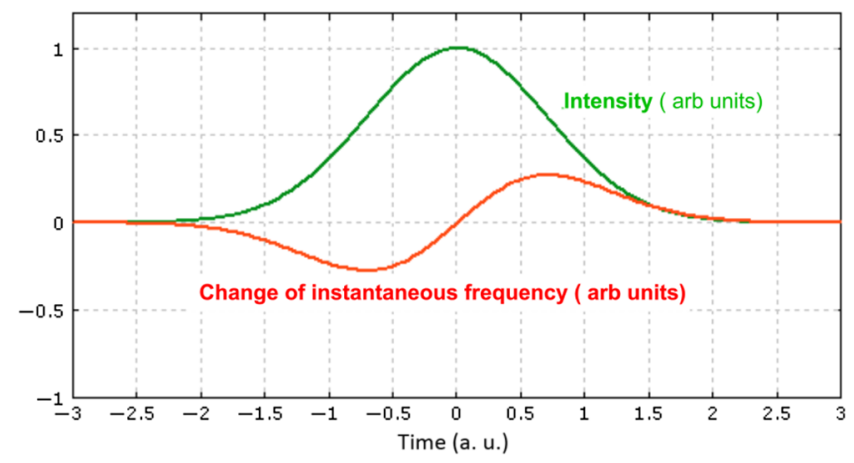

Fig. 2 The instantaneous frequency shift (red) associated with a pulse (green) as a result of the intensity-dependent refractive index. Pulse intensity and spectral shift scales ( $y$ axis) are in arbitrary units. an equilibrium, which is the concept behind the optical soliton. The formation of the fundamental soliton can be described as the balance between the dispersive length $L_{\mathrm{D}}=\tau^{2} / \beta_{2}$ and the nonlinear length $L_{\mathrm{NL}}=1 / \gamma P$, where $\gamma$ is the nonlinear coefficient and $P$ is the peak power of a pulse of duration $\tau$. Hasegawa and Tappert ${ }^{14}$ first proposed and theoretically predicted the existence of solitons in optical fibers; however, it was over 7 years before Mollenauer et al. ${ }^{15}$ carried out a series definitive experiments that demonstrated the generation of the optical soliton in fibers and their unique characteristics. The delay was due to two major technological problems: in 1973, when Hasegawa's paper was published, there were no sources of pulses with durations of a few picoseconds in the spectral region above $1270 \mathrm{~nm}$, i.e., the anomalously dispersive region of silica-based fibers, and there were no low loss fibers available in this regime either! The historical development as well as the science of the optical soliton is excellently reviewed in Mollenauer and Gordon's textbook. ${ }^{16}$

The power $P_{1}$ to generate a fundamental soliton, where $N=1=\left(L_{\mathrm{D}} / L_{\mathrm{NL}}\right)^{1 / 2}$, which will propagate over the nonlinear length without change to its pulse duration is given in practical units ${ }^{16}$ by

$P_{1}=\left(\frac{1.763}{2 \pi}\right)^{2} \frac{A_{\mathrm{eff}} \lambda^{3}}{n_{2} c} \frac{D}{\tau^{2}}$,

where $A_{\text {eff }}$ is the effective core area of the fiber and all other symbols have their usual meaning as defined above. For a $1 \mathrm{ps}, 1.55-\mu \mathrm{m}$ pulse at $500-\mathrm{MHz}$ repetition rate, in a standard fiber with a group delay dispersion of $5 \mathrm{ps} / \mathrm{nm} \mathrm{km}$, an average power of $\sim 5 \mathrm{~mW}$ is required to generate a train of fundamental solitons. If the launched input power $P$ is not exactly that of a fundamental soliton defined by $P_{1}$ above, a fundamental soliton can still evolve so long as $0.25<P / P_{1}<2.25$. The pulse simply readjusts to become a fundamental soliton by the shedding of radiation and self-adjustment of its duration. An obvious disadvantage of utilizing solitons in laser systems is that the pulse power is fixed and average power scaling can be obtained only by external amplification in chirp pulse amplification schemes. ${ }^{17,18}$ Amplification needs to be carried out over a gain length substantially shorter than the characteristic soliton or nonlinear length, or else the solitons will respond to the perturbation by shedding energy and a change in pulse duration. The soliton "area" is a conserved quantity, with the amplitude-duration product being a constant. The duration of a soliton $\tau$ is proportional to $\mathrm{D} / \mathrm{E}$, where $\mathrm{E}$ is the energy of the soliton. It can be seen that if $\mathrm{E}$ increases, $\tau$ correspondingly decreases. In an alternative amplification regime, if the gain is adiabatically applied, such that the gain per soliton length is less than about $10 \%$, then the soliton will slowly adjust its duration without shedding dispersive radiation. As the pulse continues to compress, however, instabilities from high-order dispersion and from self-effects, such as Raman gain lead to spectral shifting, pulse broadening, and the generation of dispersive radiation in the system.

Higher order soliton solutions of the nonlinear Schrödinger equation that describe soliton propagation in fibers were shown to be possible, ${ }^{19}$ where the power $P_{N}$ in the soliton of order $N$ is given by $P_{N}=N^{2} P_{1}$ and these are just a nonlinear superposition of $N$ fundamental solitons. They are generated by simply providing the required peak power at launch. On propagation, they exhibit significant pulse narrowing and periodic splitting, which results from the periodic interference between the 
individual solitons. This can lead to extreme pulse compression, which has been shown to give an optimized compression ratio of $4.1 \mathrm{~N}$. Associated with this extreme narrowing is a spectral broadening, an effective supercontinuum generation. In the 1980 s, the technique was extensively investigated as a means of ultrashort femtosecond generation of few cycle pulses. ${ }^{15,20-}$ ${ }^{22}$ Figure 3 shows a typical spectrum, on a logarithmic intensity scale, and an associated autocorrelation trace of an 18-fs pulse generated at a central wavelength of $1.319 \mu \mathrm{m}$ from an optimized compression of a high-order soliton generated by a fiber grating pulse compressor-optical fiber cascade using initial 100-ps pulses from a mode-locked Nd:YAG laser.

As noted above, although lower order picosecond solitons exhibit a periodic breathing of soliton splitting and recovery, very high-order femtosecond solitons are particularly unstable to the effects of high-order dispersion, self-interaction and noise, leading to fragmentation following the point of extreme compression. However, by terminating the propagation at the point of optimum compression, the technique does present a method for the production of few cycle pulses.

\subsection{Four Wave Mixing}

FWM and also the closely related process of modulational instability (MI), which is effectively restricted to the region of anomalous dispersion and will be described below, are of primary importance to the supercontinuum generation process, but also as will be discussed later provides a route to wavelength tunable, short pulse sources. The technique of FWM allows the generation of new frequencies in spectral regions distinct from the pump pulse wavelengths. The process can be understood as a coupling of four waves through the real part of the third order susceptibility $\chi_{3}$. Two of the waves are the pumps at designated frequencies $\omega_{1}$ and $\omega_{2}$ with these generating Stokes and antiStokes frequencies at $\omega_{3}$ and $\omega_{4}$. The process is restricted by energy and momentum considerations such that

$\omega_{1}+\omega_{2}=\omega_{3}+\omega_{4}$,

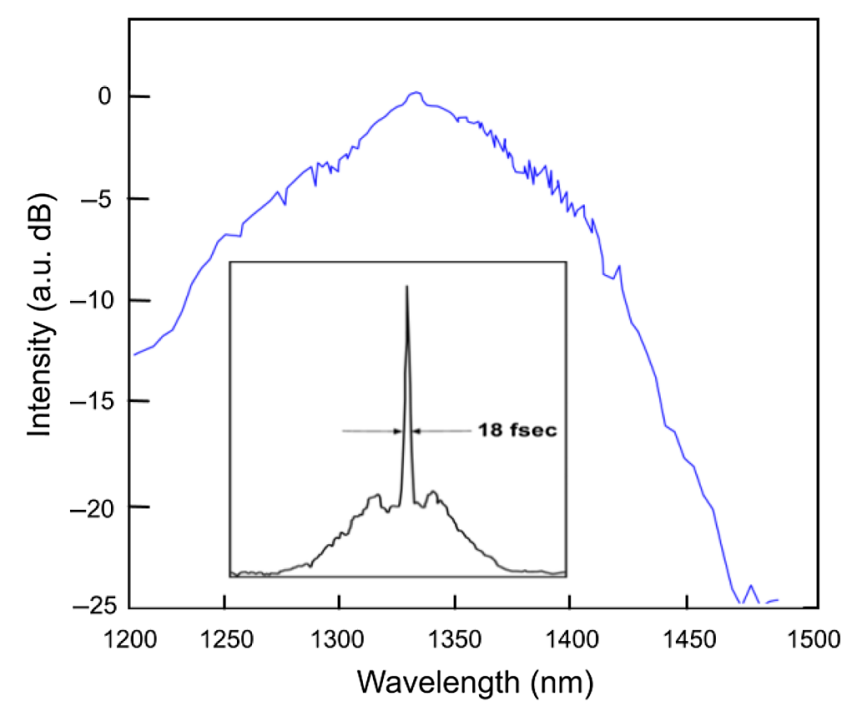

Fig. 3 Spectrum, on a logarithmic intensity scale, of an 18-fs pulse, with the corresponding temporal autocorrelation trace shown as the inset, generated through high-order soliton compression. and for efficient conversion, the phase matching condition requires that the phase mismatch of the components is zero, such that

$\Delta k=\beta\left(\omega_{1}\right)+\beta\left(\omega_{2}\right)-\beta\left(\omega_{3}\right)-\beta\left(\omega_{4}\right)+2 \gamma P_{0}$,

where the last term takes account of the nonlinear phase shift, resulting from the nonlinear refractive index, where $\gamma$ is the nonlinear coefficient and $P_{0}$ is the pump pulse power. Stolen et al. ${ }^{23}$ reported the first observation of FWM in fiber. The most commonly encountered case is where there is degeneracy of the pump such that, $\omega_{1}=\omega_{2}$. FWM can be seeded from noise; alternatively, amplification of a Stokes or anti-Stokes signal copropagating with the pump can take place, with the corresponding anti-Stokes or Stokes created so as to meet the phasematching condition and conserve energy. The FWM process when precisely phase matched exhibits the highest gain of all fiber-based nonlinearities, with the exception of stimulated Brillouin scattering; however, the latter process cannot be effectively deployed as a source of efficient ultrashort pulse generation.

Pumping in the normally dispersive regime, it is relatively easy to satisfy the phase matching equation above, particularly when pumping near the zero dispersion wavelength. Figure 4, below, shows a representative phase matching curve for a conventionally structured single-mode fiber with a dispersion zero around $1318 \mathrm{~nm}$. When pulse pumped at $1312.5 \mathrm{~nm}$, phase matching leads to the efficient generation of Stokes and antiStokes components at 1520 and $1160 \mathrm{~nm}$, respectively.

With reference to Fig. 4, it can be seen that modest tuning of the pump pulse, i.e., variation in the $x$-axis, can lead to extensive tunability of the Stokes and corresponding anti-Stokes components. From Fig. 4, it can also be seen that operation in the visible part of the spectrum would not be achieved using this fiber. In order to operate close to the dispersion zero and generate visible wavelengths, it is necessary to use PCFs, ${ }^{6-8}$ where the fiber structure can be modified to exhibit effectively endlessly singlemode operation, and a dispersion zero at any wavelength in the visible spectral range, while conventionally structured, silica-based fibers cannot operate with a dispersion zero below $1270 \mathrm{~nm}$.

\subsection{Modulational Instability}

Operating in the anomalously dispersive regime, the MI process is closely related to FWM and soliton generation and results

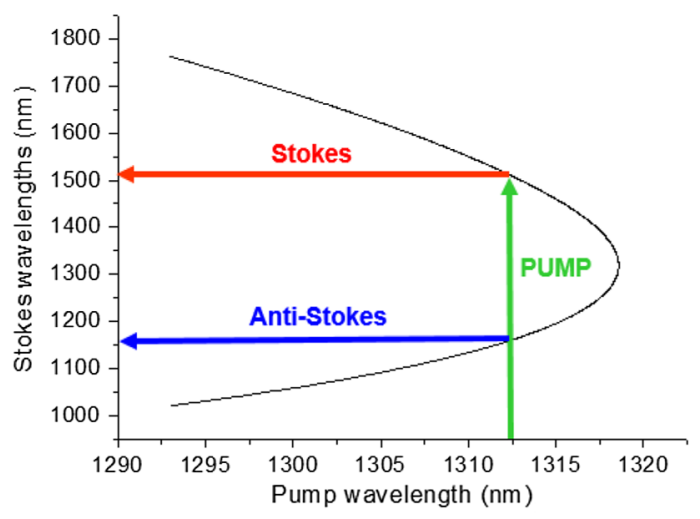

Fig. 4 The phase matched FWM process with a representative phase matching curve of a single-mode fiber with a dispersion zero at $1318 \mathrm{~nm}$, pumped at $1312.5 \mathrm{~nm}$. 
from the interplay between anomalous dispersion and the intensity-dependent refractive index. A large number of nonlinear systems exhibit such instability, evidenced by the growth of perturbations or modulations from the steady state, and have been observed in plasmas and in water waves. Hasegawa and Brinkman ${ }^{24}$ first described the process in optical fibers showing that amplitude or phase modulations on an effective CW background grew exponentially, accompanied by sideband evolution at a frequency separation from the carrier frequency that was proportional to the optical pump power. The process was first experimentally observed in optical fiber by Tai et al., ${ }^{25}$ where picosecond scale modulations were generated on the effectively CW pump background arising from 100-ps pump pulses. Using a CW pump laser, the first observations of $\mathrm{MI}$ in conventional fiber were reported by Itoh et al. ${ }^{26}$

For exponential growth, the sideband frequency separation from the carrier should be less than a critical frequency given by $\left(4 \gamma P_{0} / \beta_{2}\right)^{1 / 2}$ and the maximum growth occurring at a frequency shift of $\left(2 \gamma P_{0} / \beta_{2}\right)^{1 / 2}$, where the symbols have their meaning as previously defined. MI can be thought of as a FWM process, phase matched through SPM, with the growth of the Stokes and anti-Stokes sidebands taking place at the expense of two photons from the carrier pump. Most commonly, MI is self-starting from noise at a frequency separation around the maximum gain. It is, however, possible to initiate the process, induced MI, by seeding a signal that lies within the gain bandwidth as described above. When this process is employed, ${ }^{27}$ rapid evolution from MI sideband to solitons enhanced by the Raman gain process (see below) takes place, followed by self-Raman interaction of the generated pulses that give rise to a spectral supercontinuum on the Stokes side of the initial carrier/pump. Cross-phase modulation can also be used to induce MI on weak signals in the anomalously dispersive regime by enhancement through the group velocity matching of an intense pump signal in the normally dispersive region. ${ }^{28}$ Although this technique has been successfully used to generate 130 -fs pulses at repetition rates up to $2 \mathrm{THz},{ }^{29}$ the quality of the pulses is quite poor with the high repetition rate pulses lying on a high intensity background associated with the 100-ps pump pulses. The quality of the pulses can be improved by using feedback in laser configurations for the evolution of modulation instability ${ }^{30}$ and although refined schemes have demonstrated rates of up to $115 \mathrm{GHz}$ and pulse durations of $1.6 \mathrm{ps},{ }^{31}$ limited selectivity, both spectrally and temporally, and the presence of a low level pedestal make it difficult to compete with simpler routes to versatile short pulse generation in fiberbased assemblies and would inhibit MI laser applications, particularly, in biophotonics. However, the MI process plays a vital role in the initiation of short pulse soliton formation under single-pass picosecond and femtosecond pumping schemes that eventually leads to supercontinuum generation in fiber, a technique that provides tunable short pulse generation, albeit of questionable quality in certain circumstances.

\subsection{Raman Scattering in Silica Fibers}

The inelastic process of Raman scattering can be considered through the interaction of an incident pump photon with the internal vibrations or rotational motions, via phonons, of the glassy material of a fiber. The pump field is absorbed, exciting the molecular resonances mediated by the imaginary part of $\chi_{3}$ and this leads to the emission of a frequency down-shifted signal. The associated energy level diagram of the simple process

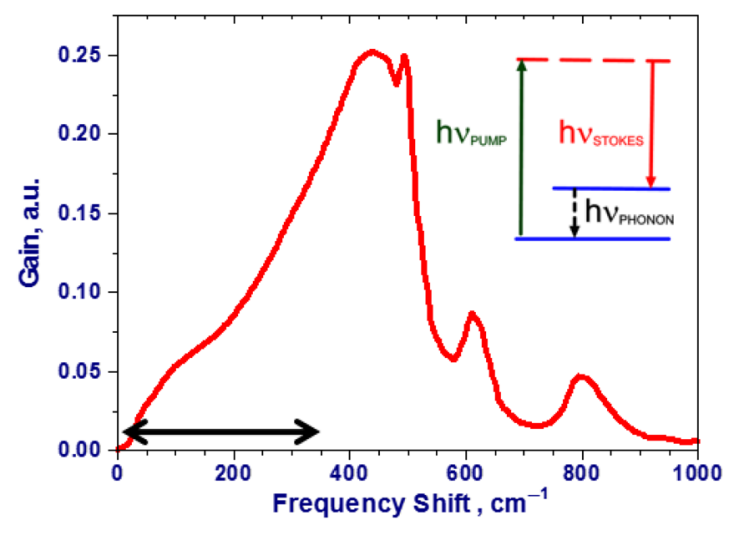

Fig. 5 The Raman gain curve for fused silica, after Stolen and Ippen. ${ }^{34}$ Inset shows the energy levels associated with the Stokes generation process through scattering off a virtual level. The arrowed line to the left indicates the representative bandwidth of a 100-fs pulse.

can be seen as the inset to Fig. 5. For an incoming pump frequency of $\omega_{P}$, the frequency of the generated Stokes (downshifted) radiation $\omega_{S}$ is given by $\omega_{S}=\omega_{P}-\omega$, where $\omega$ is the frequency of the phonons associated with the vibrational/ rotational de-excitation channel. The process can be stimulated, as was first observed in fiber by Stolen et al., ${ }^{32}$ through a coherent coupling of the pump and Stokes fields. Significant power transfer to the Stokes field can take place and conversion efficiencies of $70 \%$ are not uncommon in fiber Raman lasers, based on a simple pump geometry of a fiber laser pumping a Raman gain fiber in a basic fiber laser cavity formed by two integrated fiber Bragg reflectors at the desired Raman wavelength. ${ }^{33}$ In principle, the anti-Stokes wavelength can be generated through the absorption of energy from an oscillating excited molecule, but the signal is significantly weaker. This is because the molecule must be in the excited state and this is energetically less likely than being in the ground state. In addition, phase matching cannot be achieved in the collinear case in an optical fiber and, consequently, the Stokes process dominates and can cascade through many orders of the scattering process, providing an efficient wavelength versatile frequency down-shifted source from the pump.

As no regular structure exists in a glass, the randomly oriented molecules give rise to a broad, smooth Raman gain profile, as shown in Fig. 5, as first determined by Stolen and Ippen. ${ }^{34}$ The peak of the gain occurs for a shift of $\sim 13 \mathrm{THz}$ $\left(\sim 440 \mathrm{~cm}^{-1}\right)$ and is $\sim 40 \mathrm{THz}$ wide and, hence, is capable of supporting femtosecond pulses. When pumped around $1064 \mathrm{~nm}$, the first Stokes component occurs around $1120 \mathrm{~nm}$ and cascading can take place through second, third, and fourth Stokes components, and so on, at 1180, 1240, and $1310 \mathrm{~nm}$, respectively. ${ }^{35}$ Within each order, significant tunability is also possible, of the order of 40 to $60 \mathrm{~nm}$ depending on the pump wavelength. Consequently, gain is achievable at any wavelength, supportive of femtosecond pulse generation, and is particularly attractive since the Stokes signals generated require no waveguide phase matching.

The Raman gain process also gives rise to a peculiar process associated with optical solitons, which can be used to derive a source of broadly tunable subpicosecond pulses. If one considers the bandwidth of a short, say 100-fs pulse, as shown as the arrow headed insert in Fig. 4, it can be seen that at a shift of $10 \mathrm{THz}$, i.e., the long wavelength side of the pulse, significant 
Raman gain can be obtained from the component to the short wavelength side of the pulse. The solitons are quite robust to this gain process and on propagation, the central wavelength of the soliton continuously shifts to longer wavelength. Generally, the process tends to be self-terminating, in that with increasing dispersion with increasing wavelength, usually experienced in conventional fiber structures, the power requirements to maintain soliton properties increase and so cannot be sustained. Originally termed self-Raman interaction, the process was reported by the Dianov group ${ }^{36}$ and was later rediscovered by Mitschke and Mollenauer ${ }^{37}$ and renamed the soliton self-frequency shift. Gordon ${ }^{38}$ undertook the theoretical modeling of the process and showed that frequency shift was proportional to the inverse fourth power of the soliton pulse duration and the interaction fiber length. The process also plays a key role in the long-wavelength extension/generation mechanism in supercontinuum generation.

\section{Supercontinuum Sources}

With the development of passive-mode locking techniques and the correspondingly high-electric field strength of the so-generated pulses, anomalous spectral broadening was observed in numerous laser systems. In addition, as a result of spatial instabilities in beam profiles, self-focusing led to irreproducible filamentation, which together with the associated SPM, led to dramatic spectral broadening, a process that in laser amplifiers often caused catastrophic damage. In 1970, in a series of experiments, where by focusing the output from a frequency doubled $\mathrm{Nd}$ :glass laser into borosilicate glass and other solid and liquid samples, Alfano and Shapiro ${ }^{39,40}$ reported what was effectively the first study of supercontinuum generation. A "white-light" source covering the spectral range from 400 to $700 \mathrm{~nm}$ and with picosecond duration was recorded, with the investigators immediately recognizing the potential of the source in transient absorption spectroscopy. ${ }^{41}$

Throughout the 1970s and '80s, the simple expedient of focusing the output from a short-pulse laser source into a liquid filled cell or jet allowed the production of white light continua, with SPM identified as the major contributing nonlinear generation mechanism. As a result, time-resolved spectroscopy with spectral selection from the continuum and resolution in the femtosecond regime was undertaken. ${ }^{42}$ One problem was that regenerative amplification of the femtosecond pump pulses was needed to achieve the peak power levels adequate for continuum generation and often such open beam path, unwieldy configurations were unstable, generated irreproducible spectra and were unsuited for turn-key operation.

By the early '70s, driven by the prospect of bandwidth expansion in telecommunications through the use of optical fibers, extended lengths of low-loss silica-based fibers were becoming available. As noted in Sec. 2 above, an enormous advantage is gained through nonlinear generation in single-mode fiber geometries. As a consequence, the first fiber-based supercontinuum was reported by Lin and Stolen ${ }^{43}$ pumped by a pulsed dye laser in the visible, followed by extension of the technique using a Q-switched or mode-locked Nd:YAG laser to produce infrared spanning supercontinua of nanosecond and picosecond duration, respectively. ${ }^{44,35}$ The simple experimental configurations mirrored earlier systems with the radiation from the large-frame lasers simply lens coupled into the optical fibers. During the 80 s, it was established that soliton dynamics played a vital role in the long wavelength extension of supercontinuum generation in fiber ${ }^{45}$ and by pumping in the region of the zero dispersion, the binding through cross-phase modulation of selffrequency shifting solitons in the anomalously dispersive regime and dispersive waves in the normally dispersion region, respectively, gave rise to long and short wavelength extension of the generated spectra. ${ }^{46}$ The use of conventional single-mode fibers meant that it was difficult to achieve efficient generation of visible components in the supercontinuum spectrum since pumping in the region of the zero dispersion required laser sources above $1270 \mathrm{~nm}$ and readily available commercial ultrafast sources, such as the mode-locked Ti:sapphire laser operating around $800 \mathrm{~nm}$, could not be efficiently deployed. With the introduction of photonic crystal or holey fibers, ${ }^{6,7}$ where by manipulating the hole diameter and pitch, high modal confinement along with selection of the zero dispersion wavelength at any wavelength was achieved, hence allowing soliton generation even at visible wavelengths. Supercontinuum generation was rejuvenated and brought into an era of practical realization following Ranka's report of continuum generation covering the spectral region from 400 to $1600 \mathrm{~nm}$ pumped by a femtosecond Ti:sapphire laser. ${ }^{47}$ However, the experimental configuration simply employed bulk lens coupling and the average spectral power density of the continuum components was $\sim 10$ to $20 \mu \mathrm{W} / \mathrm{nm}$ and was relatively impractical for routine integration into diagnostic equipment.

Advances in short-pulse fiber laser technology and highpower fiber amplifiers, fuelled by multiclad gain fibers, highpower single-stripe pump diodes and novel multimode fiberbased coupling arrangements led to fully fiber integrated, high average power, small footprint, turn-key picosecond, MOPFA assemblies. Through the fusion splicing of a photonic crystal fiber, with a zero dispersion around $1040 \mathrm{~nm}$ to the output of a Yb-based MOPFA, supercontinuum generation was observed from 525 to $1800 \mathrm{~nm}$ at watts level average power and with an average spectral power density of $>1 \mathrm{~mW} / \mathrm{nm}$ throughout the continuum. ${ }^{48}$ Over the years, the average power in the supercontinuum using similar schemes has been scaled to above $50 \mathrm{~W}^{49,50}$ and there is no reason that the average power scaling could not be extended well into the several $100 \mathrm{~W}$ regime. ${ }^{51}$ The fully fiber integrated supercontinuum source has established itself as a scientific and commercial success and is routinely used in various biophotonic applications. ${ }^{52}$ Through spectral filtering from the continuum, tunable picosecond pulse sources can be derived with average powers in the $\mathrm{mW} / \mathrm{nm}$ regime; however, some caution should be extended as to the quality of the pulses so generated.

\subsection{Picosecond Pulse Pumped Supercontinuum Sources}

The power scaling in MOPFA configurations of picosecond pulses from seed lasers to average powers of many tens of watts without severe nonlinear degradation allows high-average power supercontinuum sources to be realized that effectively cover the complete window of transmission of optical fiber, from about 300 to $2300 \mathrm{~nm}$, with average spectral power densities in the region of milliwatts per nanometer. In order to optimize the supercontinuum generation process, it is advantageous to configure the system such that the pump wavelength lies in the region of anomalous dispersion and close to the zero dispersion wavelength. From Sec. 2.2, it was seen that the fundamental soliton power is proportional to $\tau^{-2}$. Consequently, for picosecond pulses from MOPFA pumps, it may naively be 
thought that high-order soliton dynamics with extreme pulse compression may play a significant role in supercontinuum generation. This is not the case. As the characteristic nonlinear length scales as $\tau^{2}$, for pulses of tens of picoseconds, the nonlinear length becomes many kilometers long, while the supercontinuum generating fiber required tends to be only a few meters.

If one considers pulses of a few picoseconds, say around $1060 \mathrm{~nm}$ with a peak power of about $10 \mathrm{~kW}$ launched into a PCF with a zero dispersion at $1040 \mathrm{~nm}$, small amplitude or phase perturbations on the pulse will experience MI. This leads to the rapid temporal breakup of the long input pulses into femtosecond scale subpulses that are amplified to soliton powers. Noise seeding plays a very significant role in the generation process. In the early stages of generation, the processes of FWM and MI dominate. Once fundamental soliton structures have evolved, soliton self-frequency shifting, together with soliton collisions further extend the supercontinuum spectrum to longer wavelengths. For solitons generated in the region of the dispersion zero, radiation extending into the normally dispersive region experiences, through cross-phase modulation, coupling with the self-frequency shifting soliton component, leading to the extension of the short and long wavelengths of the supercontinuum. Figure 6 shows a spectrogram of a continuum generated in $0.5 \mathrm{~m}$ of fiber for $10-\mathrm{kW}$ peak power pump pulses. There is a clear coupling recorded between the self-shifting soliton components and the dispersive radiation. What is also notable is the structuring of the continuum both in time and in spectrum. It should be remembered that Fig. 6 is the temporal output and the spectra associated with a single pump pulse. Most users are more familiar with extremely smooth supercontinua that represent the time integration of many millions of such spectra arising from high-repetition rate mode-locked laser pump sources. In the time domain, the supercontinuum exhibits a noise like structure with intense peaks associated with fundamental femtosecond solitons of various durations separated randomly in time representative of the noise processes driving the generation process. From pump pulse to pump pulse, the temporal structure is completely irreproducible.

Spectral filtering from such a supercontinuum does allow the selection of frequency tunable radiation; however, the temporal quality of such a generated signal can be quite poor as

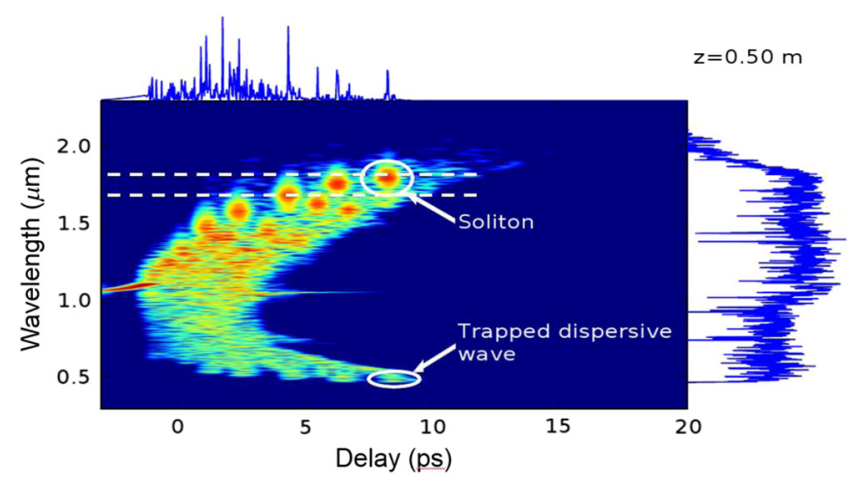

Fig. 6 Spectrogram of a supercontinuum generated in a $0.5-\mathrm{m}$ long PCF with a dispersion zero at $1040 \mathrm{~nm}$ pumped by 5 -ps pulses of 10$\mathrm{kW}$ peak power at $1060 \mathrm{~nm}$. The temporal output is shown on top and the associated spectrum is on the right. The process of soliton-dispersive wave binding is highlighted. The dashed lines represent spectral filtering to obtain a wavelength tunable pulse source. represented both by the temporal signature of the overall signal and also with reference to the temporal region defined by the dashed lines in Fig. 6. It can be seen that around $1700 \mathrm{~nm}$, the selected signal would exhibit structure dominated by intense soliton structures of differing intensities with dispersive radiation between these. From pump pulse to pump pulse, since the generation process is noise driven, the outputs are noise like, although the duration of the overall time window of the selected signal is approximately that of the pump pulse. Consequently, care should be taken in using such driven supercontinua as sources of tunable picosecond radiation if the application demands pulses of known intensity and phase structure.

\subsection{Femtosecond Pulse Pumped Supercontinuum Sources}

With the introduction of photonic crystal fibers, the dispersion zero wavelength could be manipulated such that soliton behavior could be achieved with the more common, commercially available, ultrashort pulse laser sources. This was best exemplified by Ranka's report (48) of supercontinuum generation in a $75-\mathrm{cm}$ long PCF with a dispersion zero around $770 \mathrm{~nm}$, which was pumped by the 100-fs pulses from a mode-locked Ti:sapphire laser at $790 \mathrm{~nm}$. With a peak pump power of $\sim 8 \mathrm{~kW}$, the dynamics of high-order soliton pulse compression with a corresponding spectral broadening dominated the early stages of supercontinuum generation in the fiber, ${ }^{53}$ with the optimal compression taking place over a fiber length given approximately by $\tau_{0}^{2} / N \beta_{2}$, where $N$ is the soliton order. All the processes contributing to high-order soliton compression and supercontinuum generation in photonic crystal fibers with high-power femtosecond pumping are now well understood and have been comprehensively modeled giving a remarkable agreement between theoretical prediction and experiment. ${ }^{54-57}$ At the point of extreme compression, inherent system perturbations, such as higher order dispersion and self-effects, such as stimulated Raman generation and modulational instabilities, inhibit the simple periodic soliton breathing behavior that is observed with lower order picosecond solitons. As a result, the pulse breaks apart, generating a number of fundamental solitons and dispersive radiation. The supercontinuum spectrum is further complicated by soliton collisions and self-Raman generation. In addition, the noise generated in the system through

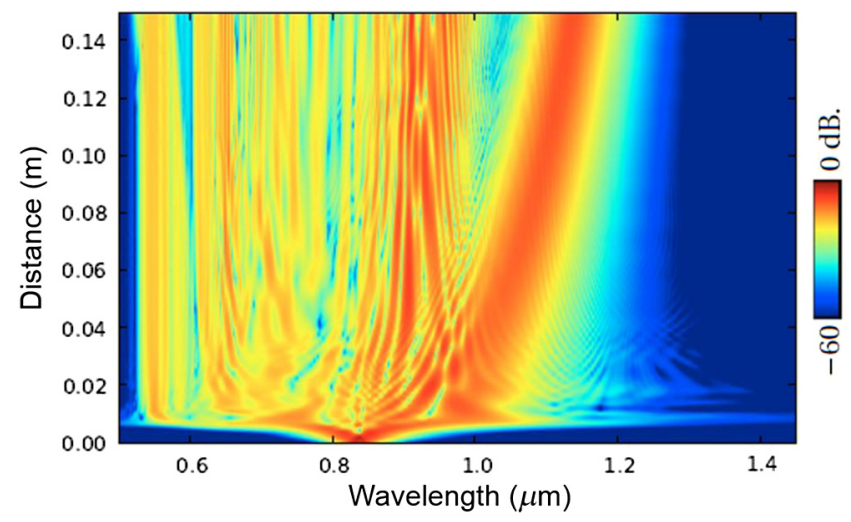

Fig. 7 Supercontinuum development along the length of a PCF with a dispersion zero around $800 \mathrm{~nm}$, pumped by 50 -fs pulses at $820 \mathrm{~nm}$ with a peak power of $10 \mathrm{~kW}$. Following initial temporal compression (spectral broadening), the pulse can be seen breaking up into numerous fundamental solitons and dispersive waves. 
the breakup of the solitons and its seeding of MI and Raman gain play an important role. As described above, soliton-dispersive wave interaction and the soliton self-frequency shift can lead to further spectral broadening as well as FWM and cross-phase modulation contributing to the generated supercontinuum.

Figure 7 shows a simulation of the spectral broadening experienced along a sample of PCF with a dispersion zero around $800 \mathrm{~nm}$ pumped by a $10-\mathrm{kW}, 50-\mathrm{fs}$ pulse at $820 \mathrm{~nm}$. Following the rapid high-order soliton compression that occurs within the first centimeter of fiber, the break-up into numerous soliton components, the generation of dispersive radiation and self-frequency shifting solitons are all in evidence after the initial temporal compression and associated spectral broadening.

Because of the very smooth profiles of supercontinua generated under femtosecond pumping from mode locked laser sources, which result from the integration of many millions of spectra, the important role played by noise is often overlooked. The spectra exhibit poor reproducibility from shot to shot and reduced coherence. The role of noise manifests itself in the process of "rogue wave" generation ${ }^{58,59}$ characterized by an excessive spectral red shift in the generated supercontinua.

Because of the noise and instability problems, if a femtosecond continuum is to be used as a source of tunable pulses through spectral filtering, it is best to operate with fiber lengths that are the optimum for high-order compression and to extract the spectra at that point. Beyond that, temporal jitter, noise, and irreproducibility degrade the temporal structure. Alternatively, soliton behavior can be completely avoided by operating solely with the pump in the normally dispersive regime, such that SPM dominates. ${ }^{60,61}$ In this case, SPM plus normal dispersion linearize the chirp. Sequential spectral selection can be used together with external dispersion compensation from, for example, a grating pair, simulating the concept of fiber grating-pair pulse compressors to provide a spectrally and temporally tunable source.

\section{Passively Mode-Locked Fiber Lasers}

Although spectral filtration of supercontinuum sources can provide a source of wavelength selectable picosecond and femtosecond pulses, the quality of the pulses so generated, as we have seen above, is not particularly good, generally resembling a short-pulse envelope of noise bursts. In addition, the average power from such sources is also relatively low. For example, with an average pump power of $\sim 20 \mathrm{~W}$ from a picosecond $\mathrm{Yb}$ MOPFA, a supercontinuum covering the spectral range from 300 to $2300 \mathrm{~nm}$ is possible with a spectral power density of about $5 \mathrm{~mW} / \mathrm{nm}$. Power scaling is possible and up to $100 \mathrm{~mW} / \mathrm{nm}$ has been produced with supercontinuum sources; ${ }^{62}$ however, industrial scale laser pumping was necessary. There is no reason that supercontinuum sources should not be power scaled to the $100 \mathrm{~s} \mathrm{~mW} / \mathrm{nm}$ but the pump source requirements, their physical size, and cost will inhibit widespread use in biophotonics laboratories. In many cases, it is more economical and energy efficient to address the pulse source requirements directly through the use of passively mode locked fiber lasers.

The process of passive mode locking of lasers was introduced by Mocker and Collins through the introduction of a liquid saturable absorber cell in a pulsed ruby laser. ${ }^{63}$ The saturable absorber, cryptocyanine in methanol, exhibited an intensity-dependent loss, with low-intensity pulses being preferentially absorbed. This periodic loss at the repetition rate of the inverse of the cavity round trip time had the effect of phase locking the gain modes of the cavity, generating a picosecond time scale pulse. In solid-state lasers, where gain saturation is insignificant, generally the generated pulse widths are of the order of the recovery time of the saturable absorber; however, where gain saturation occurs, the generated pulses can be substantially shorter than the recovery time of the absorber. With the saturation fluence being proportional to $1 / \sigma \tau$, where $\sigma$ is the absorption cross-section and $\tau$ is the recovery time, a relatively large absorption cross-section is advantageous; however, long recovery times infer long-pulse durations in systems where gain saturation is not an important process. In many solidstate schemes, saturable absorbers with dual lifetimes, where one is substantially longer than the other, can aid the pulse saturation and short-pulse shaping processes, respectively. In fibers, other processes are of significance, in particular, dispersion, where in the normal dispersion regime pulse broadening occurs or in the anomalously dispersive regime where soliton shaping leads to pulse compression.

Q-switched, mode-locked operation of a flashlamp pumped, multimode Nd-silicate glass fiber laser was reported in 1983 by Dzhibladze et al. ${ }^{64}$ Passive-mode locking was proposed through the production of saturable absorbing color centre defects generated throughout the length of the fiber, which arose via excitation by the UV component of the flashlamp in the glass host. Pulses were observed at the approximate cavity round trip time but were understandably irreproducible and of poor quality. With improved manufacturing and the availability of rare earth-doped single-mode fibers, passive mode locking of a neodymium doped system was reported using the nonlinear technique of coupled cavity mode locking, producing pulses of a few hundred picoseconds. ${ }^{65}$ Many early-mode locked fiber laser investigations utilized artificial saturable absorbers that were based upon ultrafast (femtosecond time scale) nonlinear effects in fibers, such as the nonlinear optical loop mirror ${ }^{66}$ and the improved nonlinear amplifying loop mirror (NALM) ${ }^{67}$ or nonlinear polarization rotation. ${ }^{68}$ Although the cavity configurations deploying the polarization rotation technique were a mixture of fiber and bulk components, pulses as short as $70 \mathrm{fs}$ were generated $;{ }^{69}$ however, compact, fiber integrated configurations were rapidly introduced allowing the generation of picosecond $^{70}$ and nanosecond ${ }^{71}$ pulses. Probably, the simplest all-fiber arrangement utilized a NALM in the so-called "figure of eight" configuration introduced by Duling, ${ }^{72}$ with the majority of studies based upon Er-doped schemes around $1550 \mathrm{~nm}$, where with soliton shaping, pulse durations in the femtosecond to picosecond range were readily generated. By using Raman gain, broad wavelength versatility is achievable in figure of eight lasers operating with pulse durations of the order of a few hundred femtoseconds. ${ }^{73}$ Recently, the technique has been applied to Yb-doped schemes around $1030 \mathrm{~nm}$ operating in the normally dispersive regime and pulses of a few hundred femtosecond have been produced by externally compressing the chirped pulses directly produced by the lasers, ${ }^{74}$ lending greater versatility to this relatively simple pulsed laser source. In some of the above schemes, pulse generation may not be self starting and system adjustment, and optimization is necessary to obtain mode locked operation; consequently, schemes based upon those using physical saturable absorbers have dominated the commercial market. In the past years, numerous absorbing species have been successfully employed in numerous modelocked fiber laser systems. 


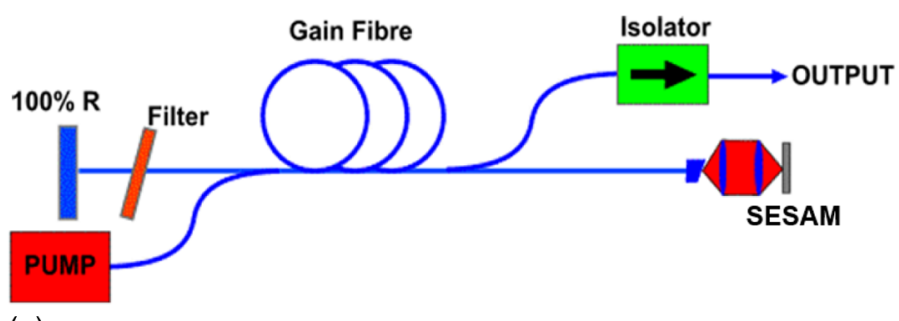

(a)

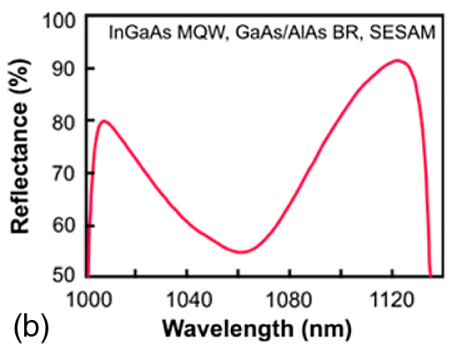

Fig. 8 (a) Schematic of a generic fiber laser mode locked using a SESAM and (b) reflection profile of a representative SESAM for operation in the 1060-nm spectral region.

\subsection{SESAM Mode-Locked Fiber Lasers}

The first application of a semiconductor saturable absorber (SESAM) to mode lock a fiber laser used a strained InGaAs/ GaAs-on-GaAs superlattice in conjunction with a diode pumped Er-doped gain medium. The sample was placed in a fiber loop configuration directly in contact with the fiber facets. ${ }^{75}$ Bulk semiconductor material InGaAsP has also been used to mode lock an Er-fiber laser, ${ }^{76}$ producing subpicosecond pulses from a linear cavity, where the saturable absorber was mounted on the output reflector and lens coupling used to focus light emerging from the fiber on to the absorber. Over the past 15 years, the semiconductor saturable absorbing mirror or SESAM, which has been successfully deployed in the passive mode locking of bulk solid-state lasers, has also been a remarkable scientific and commercial success in the mode locking of fiber lasers. By the nature of the structure of the device, it is employed as the cavity mirror in linear or Fabry-Perot type laser cavities, a schematic of which is shown in Fig. 8(a). The gain fiber is diode pumped, and an optical filter is employed intracavity both to select the bandwidth and the wavelength of the laser. The selected percentage output is taken via a fused fiber coupler and optical isolator that prevent feedback into the cavity, which would otherwise cause pulse instability. Dispersion compensation can be included in the cavity, where operation is in the normal dispersion region or when operation is in the anomalously dispersive regime, soliton shaping can lead to pulse width reduction. Coupling to the SESAM is provided by lenses or alternatively, the fiber can be butt-coupled to the SESAM facet. The SESAM consists of a semiconductor substrate on which a highly reflecting, multilayer Bragg reflector, at the laser wavelength, is placed and on top of which are the numerous alternating layers of quantum well and barriers. Figure 8(b) shows the reflection/absorption of a device consisting of alternating 5-nm InGaAs wells and 10-nm GaAlAs barriers. Typically, the SESAMs exhibit a dual recovery time, a fast subpicosecond intraband thermalization and a substantially slower $(\sim 10 \mathrm{~s} \mathrm{ps})$ carrier recombination, where the latter process helps in the initial saturation process and the faster process contributes to pulse shortening. The recovery time of the devices can be modified by ion implantation. Typically, the saturation fluence is $\sim 1$ to $10 \mu \mathrm{J} \mathrm{cm}^{-2}$, which can be readily achieved in a relatively low-power single-mode fiber laser. In addition to the complex and expensive fabrication and packaging processes, a disadvantage of the SESAM is the limited tuning or operational wavelength range of the order of tens of nanometers. Consequently, a large range of absorbers is required to service the broad range of fiber laser wavelengths.

Highly stable, self-starting, long-term mode locking of fiber lasers using SESAM technology throughout the near infrared has been achieved, generating picosecond and femtosecond pulses in Nd-doped, ${ }^{77}$ Yb-doped, ${ }^{78}$ Bi-doped, ${ }^{79}$ Er-doped, ${ }^{80}$ Tm-doped, ${ }^{81}$ and Raman gain-based ${ }^{82}$ fiber lasers.

\subsection{Carbon Nanotube Mode-Locked Fiber Lasers}

Despite the very successful application of SESAM technology to the mode-locking of fiber lasers, alternative, more versatile, single sample, solid state, fiber laser compatible sources have been sought, which were cheaper and less technologically challenging to manufacture. This was best exemplified by the carbon nanotube. ${ }^{83}$ The single-wall carbon nanotube, SWCNT, is a rolled monolayer of graphene, with diameters in the range of about a nanometer and with length scales in the micron range. SWCNTs exhibit semiconductor behavior, but this behavior is strongly determined by the unit vector of the chirality of the

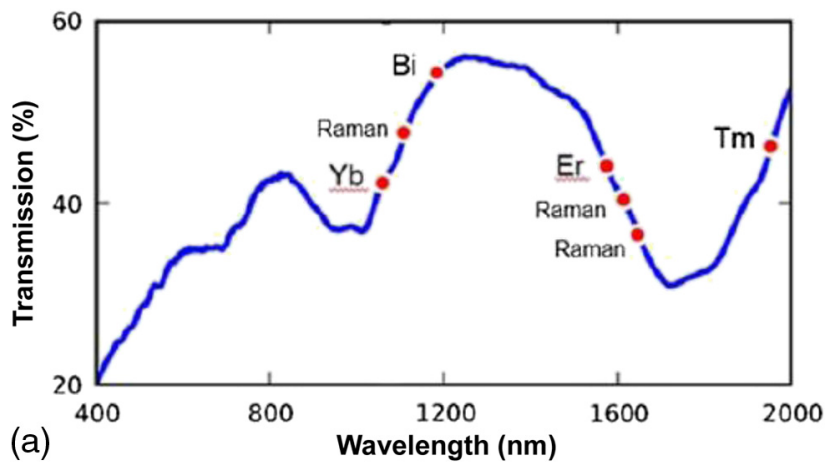

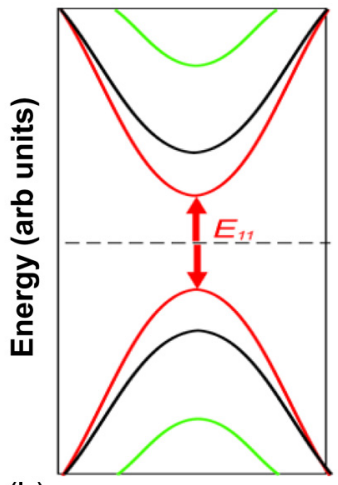

(b)

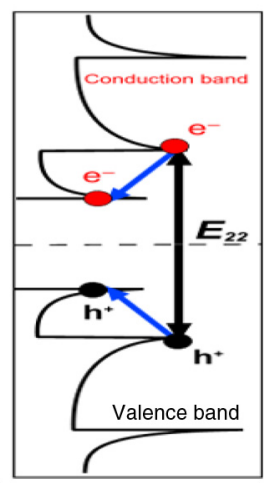

(c)
Fig. 9 (a) Transmission profile of SWCNTs embedded in a $50 \mu \mathrm{m}$ thick polymer host. The red dots on the trace indicate the different fiber laser systems that have been passively mode locked with this single sample, indicating operation over $1000 \mathrm{~nm}$. (b) A schematic band structure of a generic SWCNT. (c) A schematic of the density of states and the $E_{22}$ absorption followed by rapid relaxation to the $E_{11}$, which would be followed by recombination, is illustrated. 
folding process. Figure 9(b) is a schematic of the band structure of a generic carbon nanotube, showing an optical absorption associated with a bandgap corresponding to an energy $\mathrm{E}_{11}$ between the first valence and conduction band. Figure 9(c) is a schematic of the density of states and shows that in addition to the allowed absorption at $\mathrm{E}_{11}$, other absorptions between higher lying valence and conduction bands are allowed, for example, that corresponding to the energy gap $\mathrm{E}_{22}$. Following an absorption corresponding to $E_{22}$, rapid thermalization, of the order of tens of femtoseconds, takes place, populating the lower conduction band from which a slower, subpicosecond recombination takes place of the order of a few hundred femtoseconds. Figure 9(a) shows the absorption profile of a sample of single-carbon nanotubes embedded in a polymer host. From it, the first absorption $\mathrm{E}_{11}$ can be seen to occur at $1750 \mathrm{~nm}$, while the $E_{22}$ absorption occurs around $1000 \mathrm{~nm}$. The typical diameter $d$ for SWCNTs lies in the range from 0.7 to $1.5 \mathrm{~nm}$ and since the peak absorption wavelength $\lambda_{a}$ can be approximated by $1.49 d \mu \mathrm{m}$, where $d$ is in nanometers, then it can be seen that the principal absorption lies approximately in the range of 1 to $2 \mu \mathrm{m}$.

Carbon nanotubes can be manufactured using a variety of processes including arc discharge and laser ablation. Despite postmanufacture selection processing, a range of tube diameter distributions will exist and this gives rise to an increase in the absorption bandwidth. Manufacturing a sample with a range of tube diameters can be advantageous in that it allows a single sample to act as a saturable absorber for several different laser systems. ${ }^{84}$ Although the tubes not involved in the actual absorption process will give an increased loss due to scattering, the high gain associated with the fiber laser can readily overcome these unsaturable losses, which can be as much as a few percent. As it is difficult to manufacture tubes with subnanometer diameters, SWCNTs with fundamental absorptions in the near infrared and visible are consequently difficult to obtain; however, operation using the higher energy $\mathrm{E}_{22}$ absorption overcomes this ${ }^{85}$ at the cost of a higher saturation intensity requirement, primarily because of the reduced times scales associated with the rapid redistribution and thermalization.

The typical operational saturation intensities of carbon nanotube samples are quite similar to those of SESAMs, with a value in the region of $10 \mathrm{MW} \mathrm{cm}^{-2}$ for operation on the $\mathrm{E}_{11}$ transition, which has a typical recovery time of the order of 1 ps. Such intensities are readily achievable intracavity with single-mode fiber lasers. For operation on the $\mathrm{E}_{22}$ transition, the saturation intensity increases to $\sim 220 \mathrm{MW} \mathrm{cm}^{-2}$, indicating the role of the much faster dephasing time, which is $\sim 40$ to $50 \mathrm{fs}$, but still achievable with a single-mode fiber laser. Figure 10(a) shows a schematic of a passively mode-locked fiber laser, where the carbon nanotube saturable absorber, which is incorporated in a polymer film with a thickness of $\sim 50 \mu \mathrm{m}$, is mounted intracavity on the facet of a standard fiber coupler. The fiber gain block, which can contain the rare earths, Yb, Er, Tm, or can be based upon Raman gain (but then usually with extended length) is diode laser pumped. An isolator ensures unidirectional operation and an intracavity interference filter allows bandwidth control and wavelength tunability, while polarization control is also deployed where the fiber itself is not polarization preserving. The output is taken via a fused coupler optimized for the required operational power, which is typically in the $10 \mathrm{~s} \mathrm{~mW}$ level.

Figure 10(b) shows a representative autocorrelation trace of a 750-fs pulse at $1550 \mathrm{~nm}$ generated by a SWCNT saturable absorber passively mode-locked Er fiber laser. Such a laser operates in the average soliton regime, ${ }^{86,87}$ where the overall cavity is constructed of several different fibers and components, which on average is anomalously dispersive at the operational wavelength and where the solitons so-generated take on the characteristics at this average dispersion value, despite perturbations such as loss, gain, and differing dispersion occurring at each transit of the cavity. When the characteristic nonlinear or soliton length is substantially larger than the length scale of the perturbation, then the average soliton behavior is maintained; however, when the generated pulses are short, in the picosecond or femtosecond regime, this condition for most cavity configurations is difficult to physically maintain and when the soliton length is comparable to that of the cavity length, the soliton will respond to a perturbation by shedding radiation. When this happens, spectral interference can occur as a result of the phase interference between the soliton and the dispersive radiation. This manifests itself as so-called "Kelly sidebands," ${ }^{88-90}$ which can be seen on the spectrum in Fig. 10(c). The appearance of this dispersive radiation, characterized by the sidebands and that can contain significant system energy, as much as $30 \%$ to $40 \%$, severely degrades the quality of the output pulses.

With mode-locked fiber lasers typically constructed from several meters of fiber, operational repetition rates are generally in the range of a few tens of megahertz. For some pump-probe applications, where long decay times may be investigated, the pulse repetition rates may need to be increased so that after excitation, the following pump pulse does not arrive during the investigated decay dynamics. With fiber mode locked fiber lasers, this can be readily achieved simply by adding more

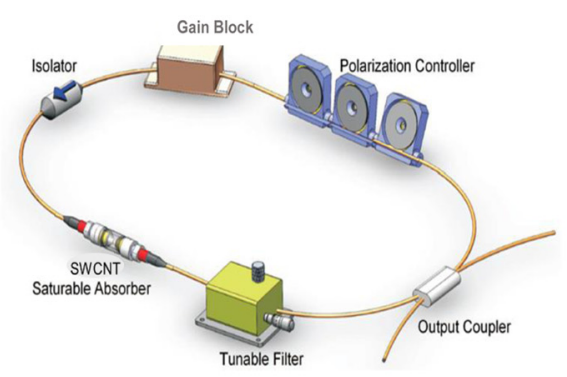

(a)

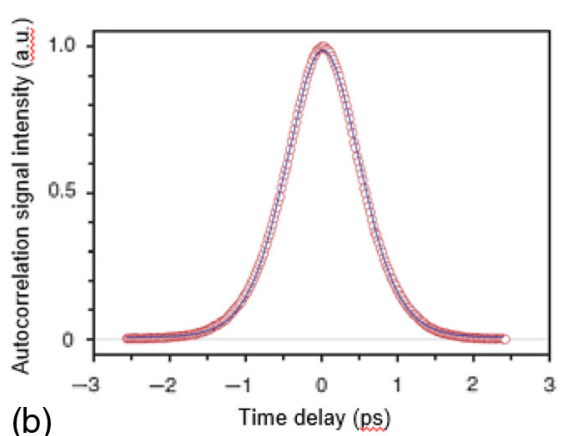

(b)

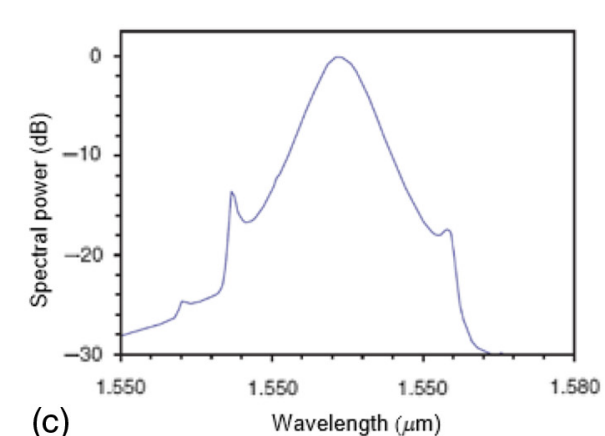

(c)

Wavelength $(\mu \mathrm{m})$

Fig. 10 (a) Schematic of a passively mode-locked fiber laser incorporating a SWCNT polymer film saturable absorber placed within the fiber coupling assembly. ( $b$ and $c$ ) A typical autocorrelation trace of a 750-fs pulse at $1550 \mathrm{~nm}$ generated by such a laser and inset is the corresponding spectrum. 
passive fiber and mode locked operation with cavities several kilometers long has been demonstrated. ${ }^{91}$ For operation in the normally dispersive regime, for example, with a Ybbased gain medium, the overall pulse generation process leads to a monotonic and positive frequency sweep (an upchirp) being generated across the pulses and with dispersion dominating the process, the generated pulses exhibit durations many times the transform-limited duration. This can be advantageous in that the pulse energy, which is unlimited as would not be the case for soliton operation, can be scaled to the microjoule level, while avoiding the problems of nonlinearity because of the extended pulse durations. Consequently, such pulses can also be used as the seed sources for fiber-based chirped pulse amplifier schemes, where external pulse compression schemes such as grating pairs can be used to recompress the pulses to the picosecond regime. Many giant chirp mode-locked fiber oscillators, however, exhibit bandwidths that are less than a nanometer. Consequently, with durations in the nanosecond regime, typical conventional grating pair separations in excess of $50 \mathrm{~m}$ may be required for pulse compression, which is quite impractical. In such circumstances, chirped fiber grating technology can be used for pulse compression, but this then limits the operational pulse energies. In a cavity similar to that of Fig. 10, Woodward et al. ${ }^{92}$ incorporated $840 \mathrm{~m}$ of single-mode fiber, generating pulses of $1.02 \mathrm{~ns}$ at a fundamental repetition rate of $244 \mathrm{kHz}$ at $1058 \mathrm{~nm}$ with a pulse energy of $\sim 1 \mathrm{~nJ}$, which exhibited the characteristic square sided spectra associated with giant chirped pulses, with a bandwidth of $0.8 \mathrm{~nm}$. On coupling from the cavity, the pulses traversed a fiber-coupled circulator incorporating a 40-cm long chirped fiber Bragg grating, which compressed the pulses by almost two orders of magnitude to $11 \mathrm{ps}$. The compressed or uncompressed pulses can be amplified and allow supercontinuum generation with picosecond and nanosecond pumping, respectively, at low $(100 \mathrm{~s} \mathrm{kHz})$ repetition rate and low average powers, of the order of a few $10 \mathrm{~s} \mathrm{~mW}$, for application in time-resolved spectroscopy.

By incorporating a chirped grating in an optical circulator assembly within the cavity, giant chirp, nanosecond scale pulses can also be generated at more conventional $(\sim 10 \mathrm{~s} \mathrm{MHz})$ repetition rates, ${ }^{93}$ while by reversing the grating, such that short wavelengths are reflected first, the net cavity dispersion can be made negative and average soliton performance obtained with the generation of pulses in the few picosecond regime. ${ }^{94}$

\subsection{Graphene Mode-Locked Fiber Lasers}

Graphene is composed of a flat single layer of carbon atoms arranged in a two-dimensional (2-D) lattice through $\mathrm{sp}^{2}$ hybridization. It is distinct from other semiconductor materials in that it exhibits a linear dispersion relationship and that this is characterized by a zero bandgap configuration such that the valence and conduction bands are represented by cones that touch at their apices, with the density of states also exhibiting a linear dependence. Surprisingly, a single monolayer of grapheme exhibits a significant absorption of $\sim 2.3 \%$ and as a result of the linear dispersion and density of states, the absorption of graphene is effectively wavelength independent. Consequently, it is attractive as a potential universal saturable absorber, applicable to any laser system. As the number of layers in graphite increases, the absorption increases linearly. Single layer graphene is commonly prepared by liquid phase exfoliation and following settlement after sonification, the solution is centrifuged and selection made from the dispersion. This is then

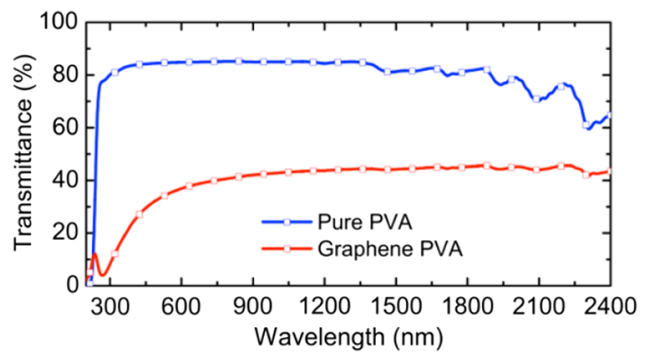

Fig. 11 Low light level transmittance of a pure PVA host sample and a graphene-PVA composite 50- $\mu \mathrm{m}$ thick saturable absorber.

added to a PVA solution and centrifuged again and laid in layers of $\sim 40$ to $50 \mu \mathrm{m}$ that can be placed on the facets of a fiber coupler, as in the case of carbon nanotubes and as illustrated in Fig. 10 above. Figure 11 shows the absorption profile of an undoped PVA sample and a graphene in PVA assembly indicating that the absorber consists of $\sim 21$ layers of graphene. The relatively featureless absorption profile illustrates the potentially extensive spectral region for saturable absorption extending throughout the complete visible to beyond $2400 \mathrm{~nm}$. In addition, graphene in PVA-based saturable absorbers also tends to exhibit reduced nonsaturable losses as compared to carbon nanotube devices.

Although graphene has a nominally wavelength-independent linear absorption, the saturable absorption behavior shows a relatively strong wavelength dependence, being substantially greater at shorter wavelengths than in the near infrared around $2 \mu \mathrm{m}$. This is a result of the band filling dynamics. Following initial absorption, the excited electrons thermalize in a time scale of 10 to 100 fs to form a Fermi-Dirac distribution, blocking some of the original interband transitions and intraband phonon scattering occurring of a time scale of a picosecond or less further cools the distribution. Following this, electron-hole recombination is the dominant relaxation process. As a result, saturation intensities are in the range of 10 to $200 \mathrm{MW} \mathrm{cm}^{-2}$, but still quite achievable with conventional single-mode fiber lasers throughout the operational bandwidth of the absorber. Bao et al. ${ }^{95}$ demonstrated the first passive mode locking of an Er-doped fiber laser, generating $\sim 1$ ps pulses of high dynamic range around $1560 \mathrm{~nm}$. Since then, the passive mode locking of $\mathrm{Yb}^{96}$ and $\mathrm{Tm}^{97}$ fiber lasers using graphene saturable absorbers has been reported, as well as numerous bulk solid state laser systems. ${ }^{98}$ Generally, the mode locked performance is relatively similar to that based upon nanotube or SESAM-based devices and like both systems, occasional damage to the substrate is observed as a result of random Q-switching, which requires realignment or replacement of the absorber.

Laser action in the near-infrared spectral regions not covered by rare earth-doped fiber amplifiers can be achieved through the use of fiber Raman geometries. The most common configuration is to use a high-power CW rare earth-doped fiber laser, most commonly an Yb-based system, to act as the fundamental pump source and to convert this to the required wavelength through a series of Raman Stokes shifts. This is most simply achieved through the use of a cascaded resonator based upon a highly nonlinear, usually germanium-doped fiber, comprising a series of nested cavities consisting of embedded fiber Bragg gratings at the desired wavelengths, as first described by Grubb et al. ${ }^{99}$ Through system refinement and development, fiber Raman lasers are currently capable of operating at many 
hundreds of watts $\mathrm{CW}$ and with conversion efficiencies approaching the limit set by the quantum conversion. ${ }^{33}$ Consequently, any wavelength in the window of transparency of the fiber can be generated through the cascaded Raman process, provided a suitable pump laser is available and this is generally achieved using a rare earth doped laser, a Raman fiber laser or their frequency doubled analogue, making a simple fiber loop a universal wavelength source. Consequently, if a universal saturable absorber can be inserted, such a simple configuration could constitute a universal pulse source, with a wavelength of operation determined solely by the pump wavelength.

Using a highly nonlinear, 100-m length of germanium-doped fiber, a Raman laser at $1666 \mathrm{~nm}$, pumped by a high-power Er fiber ASE source at $1555 \mathrm{~nm}$, has been mode locked by a mixture of $90 \%$ double wall carbon nanotubes plus $10 \%$ single wall tubes in a polymer host. The laser delivered 500-ps pulses at the fundamental cavity repetition rate of $1.72 \mathrm{MHz}$, with an average power of $0.1 \mathrm{~mW}$. The output spectrum, $1.6 \mathrm{~nm}$ wide, capable of supporting pulses of about $2 \mathrm{ps}$, exhibited the square sided characteristic associated with an inherent giant chirp. Compression was therefore undertaken using the negative dispersion of a Gedoped fiber with a dispersion zero at $1320 \mathrm{~nm}$, while simultaneously amplifying the pulses using Raman gain pumped by the residual fundamental pump power of the oscillator. ${ }^{100}$ Pulses of $2 \mathrm{ps}$ and $1.4-\mathrm{kW}$ peak power were generated. The technique was subsequently extended to other spectral regions through the use of $\mathrm{Yb}$ fiber laser pumping and a graphene saturable absorber in all-fiber cavities essentially consisting of these two components and the possibility of pulse compression demonstrated; however, these Raman gain-based, universal pulse source units are yet to be fully developed and operation outside the mid infrared remains to be reported. ${ }^{101,102}$

\subsection{Other Two-Dimensional Saturable Absorber Mode-Locked Fiber Lasers}

With the remarkable impact of graphene, not just as a saturable absorber in laser science but through the potential for wide deployment in photonic and optoelectronic devices, studies have been implemented to investigate the properties of other 2-D nanomaterials, such as the metal dichalcogenides, molybdenum disulphide $\mathrm{MoS}_{2}$ and tungsten disulphide $\mathrm{WS}_{2}$ as well as their diselenide analogues, for photonic application.

Wang et al. ${ }^{103}$ were the first to suggest that $\mathrm{MoS}_{2}$ nanosheets could be used as a saturable absorber based upon their measurements of the nonlinear transmission at $800 \mathrm{~nm}$. The band gap of an isolated monolayer of $\mathrm{MoS}_{2}$ is in the visible, around $689 \mathrm{~nm}$ $(\sim 1.8 \mathrm{ev})$ but can be altered by defect states in the material. Wang showed that both Mo and $\mathrm{S}$ defects can reduce the bandgap allowing longer wavelength to contribute to the absorption and saturation process. Increasing the $S$ defect states leads to semiconductor behavior and a substantial lowering in energy of the bandgap. Reported measurements of the saturation intensity vary between 1 and $100 \mathrm{MW} \mathrm{cm}^{-2}$ and are achievable with fiber lasers. Broadband saturable absorption in few layer $\mathrm{MoS}_{2}$ was reported by Zhang et al. ${ }^{104}$ and the material, in the form of a flake on a fiber facet, was used to mode lock an $\mathrm{Yb}$ fiber laser, generating stable 800 -ps pulse at a $6.5-\mathrm{MHz}$ repetition rate.

Like graphene- and nanotube-based devices, different techniques can be used to produce $\mathrm{MoS}_{2}$ saturable absorbers, such as solution processing, chemical vapor deposition, spray techniques, pulse laser deposition, or manufacturing thin film PVA samples of the order of $40-$ to $50-\mu \mathrm{m}$ thick containing the nanomaterial flakes. The most commonly encountered mode locked laser configuration uses the latter technique where the sample is mounted on the ferrule facet of a fiber connector and deployed, as shown in Fig. 10. As previously mentioned, this configuration is prone to damage, particularly to the host material as a result of thermal damage from high-average power operation or from spurious damage as a result of random Q-switching. Improved power handling can be achieved by using microtapered fibers or side polished D-shaped fiber that rely on evanescent wave interaction and longer interaction lengths that increase the power handling capability of the mode locked laser.

$\mathrm{MoS}_{2}$ has also been used to mode lock Tm fiber lasers ${ }^{105}$ and has achieved broadly tunable mode locked operation with an Er fiber laser. ${ }^{106}$ Figure 12 shows the tuning range obtained in a laser configuration similar to that of Fig. 10, where mode locking was achieved with $\mathrm{MoS}_{2}$ flakes placed in a PVA host. For reference, the amplified spontaneous emission spectrum of Er is also shown and on the right, a representative autocorrelation of a 960-fs pulse obtained around the center of the tuning range at $1552 \mathrm{~nm}$ is shown. Pulses as short as $637 \mathrm{fs}$ have been achieved with $\mathrm{MoS}_{2}$ mode locked Er-based fiber lasers. ${ }^{107}$

Using $\mathrm{WS}_{2}$, mode locking of $\mathrm{Er}^{108}$ and Ho- $\mathrm{Tm}^{109}$ doped fiber lasers has been successfully demonstrated, while $\mathrm{MoSe}_{2}$ has been used to produce picosecond pulses with an Er-doped fiber laser system. ${ }^{110}$ Other material structures have recently been introduced such as black phosphorous that has allowed the generation of pulses as short as $272 \mathrm{fs}$ with an Er fiber laser ${ }^{11}$ and from the new family of topological insulators that have recently been identified, that currently contain more
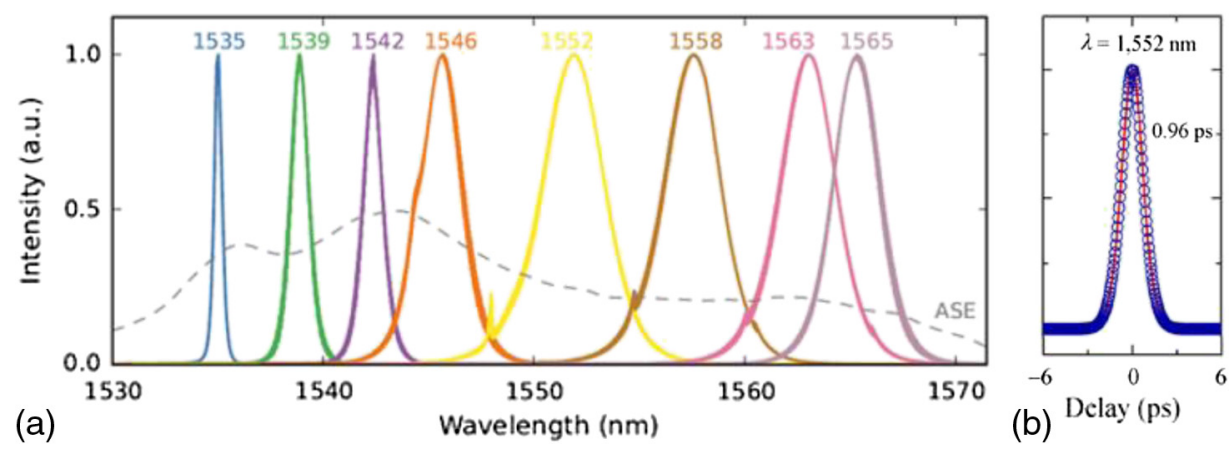

Fig. 12 (a) Tuning range obtained from an Er-doped fiber laser mode locked using a $\mathrm{MoS}_{2}$ saturable absorber. (b) A representative autocorrelation of the 960 -fs pulses obtained at $1552 \mathrm{~nm}$ with the system. 
than 30 species, $\mathrm{Bi}_{2} \mathrm{Te}_{3}, \mathrm{Bi}_{2} \mathrm{Se}_{3}$, and $\mathrm{Sb}_{2} \mathrm{Te}_{3}$ have been used to mode lock Er and Tm fiber lasers with durations down to a few hundred femtoseconds. ${ }^{112-114}$ Consequently, there is a vast array of saturable absorbing species that can be used to mode lock fiber lasers each with distinct yet quite complementary properties. Most commonly, the absorbers are deposited in a polymer host and mounted on the facet of the ferrule of a fiber connector; however, this configuration is prone to thermal and/or peak power damage problem and perhaps consideration should be given to absorbers mounted in optimized ultrathin glass hosts. ${ }^{115}$ By mounting the absorbers on tapered microfibers or on side polished fibers, longer interaction lengths can be used and since interaction is through the evanescent field, the power handling capacity significantly increases. As regards what absorber to use? - perhaps, the greatest versatility, particularly from the range of operable wavelengths, is provided by a mixture of carbon nanotubes and although this may slightly increase the nonsaturable loss, the high gain achievable with fiber lasers can readily overcome this slightly negative aspect.

\section{Sources Based Upon Adiabatic Soliton Compression}

As described in Sec. 2.2, the soliton power is proportional to $D / \tau^{2}$, where $\mathrm{D}$ is the group delay dispersion of the transmitting fiber and $\tau$ is the pulsewidth. On experiencing loss through propagation over a distance that is long compared to the characteristic soliton period $z_{0}$, given by $\pi^{2} \mathrm{c} \tau^{2} /\left[(1.763)^{2} \lambda^{2} D\right]$, solitons exhibit experience a broadening of the pulsewidth increasing exponentially with distance. ${ }^{116}$ It was suggested by Tajima ${ }^{117}$ that if the transmission fiber was constructed such that the dispersion exponentially decreased with increasing length, then the broadening associated with the propagation loss could be totally compensated and the soliton would propagate without temporal distortion. The required dispersion profile is readily manufactured directly at the drawing tower through the construction of a fiber with a core tapering along the length of the fiber with an exponential dependence, since group velocity dispersion is precisely controlled by the size of the core diameter. The operation of the tapered fiber is equivalent to providing adiabatic amplification, the action of which is to slowly compress solitons with no energy loss to dispersive waves. In their seminal work, Zabusky and Kruskal ${ }^{118}$ had shown that sinusoidal waves would evolve into solitary wave plus a dispersive wave component, while Hasegawa and Kodama ${ }^{116}$ had shown that a pulse of any reasonable shape and energy would evolve into a soliton; however, Dianov et al. ${ }^{119}$ were the first to propose the application of a dispersion decreasing optical fiber to adiabatically convert a sinusoidal optical beat signal from two closely frequency-spaced laser sources into a train of fundamental solitons that exhibited no dispersive wave component and that was experimentally realized by Mamyshev et al. ${ }^{120}$

The parameters of the dispersion decreasing tapered optical fiber must be carefully chosen to allow input pulse compression at the chosen repetition rate, while the ratio of the input to output dispersions effectively sets the compression ratio obtained. The upper repetition rate is limited by the input peak/average power requirements set by the soliton power at launch. The lower operational repetition rate is primarily determined by the fiber lengths available. Typically, for a fiber with an input dispersion of $5 \mathrm{ps} / \mathrm{nm}^{-1} \mathrm{~km}^{-1}$, at $1.55 \mu \mathrm{m}$, the characteristic length of a 10-ps soliton is around $8 \mathrm{~km}$; over this length as a typical guide line, an effective amplification of about $10 \%$ will maintain adiabatic behavior but means that several tens of kilometers of fiber are required for compression to the single picosecond regime. Chernikov et al. ${ }^{121}$ reported the first use of two CW operating semiconductor distributed feedback (DFB) lasers to generate a sinusoidal beat signal, which was amplified in an Er-doped fiber amplifier and launched into a 2.2-km long tapered fiber with a dispersion profile varying from 6.5 to $1.1 \mathrm{ps} \mathrm{nm}^{-1} \mathrm{~km}^{-1}$ used to generate output pulses from 3.0 to $1.5 \mathrm{ps}$ at corresponding repetition rates from 80 to $130 \mathrm{GHz}$. A problem with the amplification and transmission of narrow line seed sources is the generation of backward travelling stimulated Brillouin, but this can be overcome by applying a relatively low frequency modulation directly to the laser diode sources. The greatest problem to the technique, however, is the need for the specialist tapered fiber, which is expensive and complicated to manufacture as well as setting quite restricting operational limits to the compression ratios achievable and the input repetition rate of the signals.

In the theoretical modeling of the pulse propagation and compression through a taper, a split step technique can be applied where the effects of dispersion and nonlinearity and dispersion can be treated separately over a multitude of sequential steps. Experimentally, a similar method can be used, breaking the tapered profile into a series of comb-like profiles, where nonlinearity can be managed in a length of low (effectively negligible) dispersion fiber, while the dispersion decreasing profile is obtained using a fiber with high fixed dispersion and varying (decreasing) length, mimicking the net dispersion profile experienced per step, thus making up the comb profile. In addition, surprisingly few steps, as few as 6 to 10 , can give an effective adiabatic compression, generating clean pedestal free solitons from sinusoidal input signals. Experimentally, this was first realized theoretically and experimentally by Chernikov et al., ${ }^{122}$ allowing the generation of 2.2-ps solitons at $\sim 60 \mathrm{GHz}$ in a totally fiber-integrated package incorporating a dual frequency erbium fiber laser as the seed beat signal.

In a further simplification, the continuously dispersion decreasing profile of a long length tapered fiber was experimentally simulated by a series of six fibers of differing dispersions and lengths in a step-like profile. This allowed the generation of 670 -fs pulses at $\sim 100-\mathrm{GHz}$ repetition rate for input average powers $\sim 400 \mathrm{~mW}$ from an amplifier dual frequency source based upon DFB semiconductor lasers at $1.53 \mu \mathrm{m}^{123}$ and on increasing the input power to $800 \mathrm{~mW}$ pulses of $\sim 500 \mathrm{fs}$ were achieved.

Although these novel composite fiber profiles successfully mimicked and replaced the more experimentally taxing to manufacture tapered fibers, considerable care had to be placed in the assembly of the step and comb-like configurations to minimize splice loss between the successive elements. In addition, compression ratios were fixed by the characteristic input and output dispersions, with the operational repetition rate also restricted. A simpler solution is provided by deploying real distributed amplification and this is achieved using Raman gain in what is effectively conventional silica fiber of fixed dispersion in a counter propagating pump geometry. Figure 5 showed the stimulated Raman gain profile of silica fiber, which has a peak gain coefficient of about $10^{-13} \mathrm{~m} / \mathrm{W}$ and a gain bandwidth capable of supporting sub-100 fs pulses. A distinct advantage of Raman gain is that gain can be achieved at any wavelength determined only by the wavelength of the pump; however, the relatively low gain 


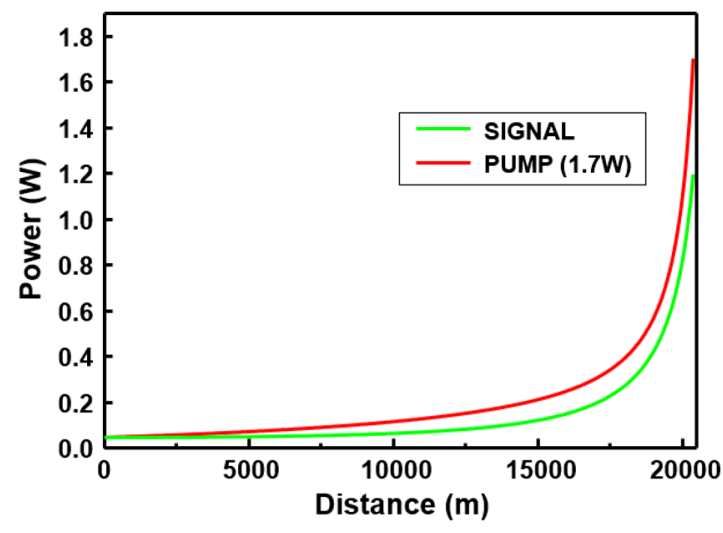

Fig. 13 Simulation of Raman gain provided by a 1.7-W CW pump at $1457 \mathrm{~nm}$ in a counter propagating pump scheme, over $20 \mathrm{~km}$ of silicabased fiber for a input signal at $1557 \mathrm{~nm}$.

coefficient of conventional silica-based fibers means that for an average pump power of around $1 \mathrm{~W}$ in order to achieve a gain of $\sim 20 \mathrm{~dB}$, fiber lengths in the range of 10 to $20 \mathrm{~km}$ need to be deployed. Figure 13 shows a simulation of the exponential gain achieved using a counter-propagating 1.7-W CW pump at $1457 \mathrm{~nm}$ providing gain for an input $10 \mathrm{ps}$ signal at $1557 \mathrm{~nm}$. At input, a 10-ps soliton has soliton period of $2.48 \mathrm{~km}$ and over that distance, the signal received a net gain of $1.7 \%$, consequently well within the guide line of $<10 \%$ gain per solition period for adiabatic gain. At output, after $\sim 20 \mathrm{~km}$ propagation, the pulse has reduced to $1 \mathrm{ps}$ with a corresponding soliton period of $24.8 \mathrm{~m}$ and received a gain of $3.4 \%$ over that final length, again within adiabatic gain limits, giving an average output power in excess of $1 \mathrm{~W}$.

Using a tunable CW diode laser and an inline electroabsorption modulator, followed by an inline Er amplifier to boost the signals to fundamental soliton power, pulses of $\sim 10 \mathrm{ps}$ were generated at $10 \mathrm{GHz}$ and launched into $21 \mathrm{~km}$ of dispersionshifted fiber, pumped in a counter-propagating geometry, by a CW fiber Raman laser at $1455 \mathrm{~nm}$ to provide adiabatic Raman gain and consequential soliton pulse narrowing. This allowed the generation of pulses as short as $540 \mathrm{fs}$, depending on the fundamental pump power, tunable from 1535 to $1560 \mathrm{~nm} .{ }^{124}$ In a relatively similar experimental configuration only deploying a Mach-Zehnder inline amplitude modulator to generate sinusoidal input signals at $20 \mathrm{GHz}$, wavelength tunable compressed pulse durations selectable from 8 ps to $450 \mathrm{fs}$ were obtained simply by varying the Raman amplifier pump power over the range of 0.2 to $1.3 \mathrm{~W} .{ }^{125}$

As the above seed sources illustrated, successful adiabatic pulse compression using Raman gain can be applied to either input pulses with sech-like or sinusoidal envelopes; however, gain lengths effectively limit the upper seed durations. It is well known that spectral windowing of self-phase modulated pulses gives rise to temporally shorter pulses, ${ }^{126}$ albeit with an associated energy loss; however, the latter can readily be overcome by inline amplification. This technique has been generalized by Mamyshev, ${ }^{127}$ through the spectral masking of a phase-modulated $\mathrm{CW}$ signal, the basis of which is shown in Fig. 14.

As illustrated by Fig. 14(a), if a phase modulator is used to impose a sinusoidal phase shift $\Delta \phi$ on a $\mathrm{CW}$ signal, this will give rise to a corresponding frequency shift $\Delta \omega$ since $d \omega=-d \phi / d t$, as shown in Fig. 14(b). If the signal is then
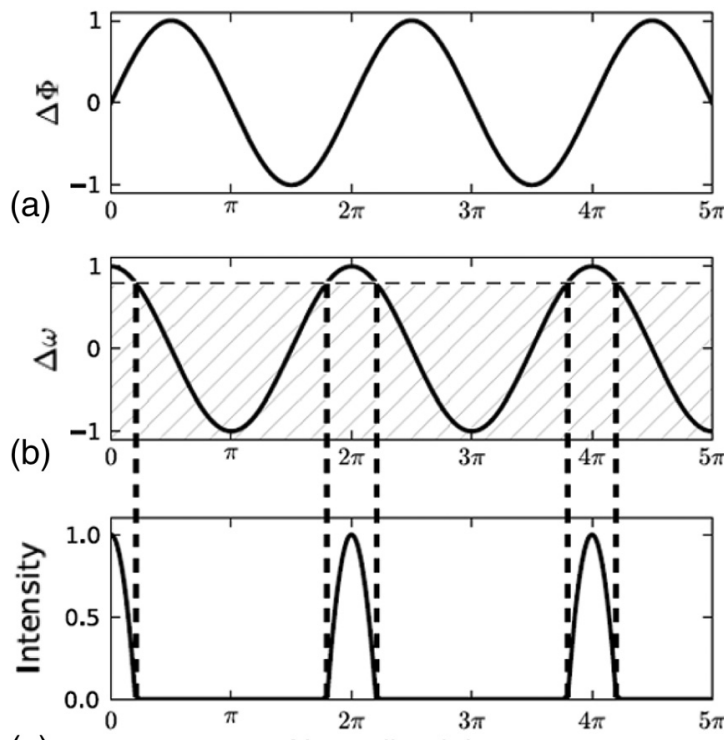

(c)

Normalised time

Fig. 14 The principle of pulse generation through spectral masking of a sinusoidally phase modulated $\mathrm{CW}$ signal, where (a) phase modulation of the signal leads to (b) an instantaneous frequency shift which on spectral filtration or removal, as shown by the shaded region, leads to (c) the temporally narrowed pulses.

passed through an optical edge filter with a high frequency transmission, represented by the hashed area in Fig. 14(b), then as shown in Figs. 14(b) and 14(c), a temporally shortened pulse at the repetition rate of the frequency modulation is obtained. The technique can be equally applied to the components at the maximum or minimum frequency excursion. At these positions, there is minimal frequency chirp. The use of a sigmoidal filter generates pulses that are closely fitting to a sech shape, although a Gaussian filter tends to reduce noise effects and gives rise to smoother generated pulses. The generation mechanism is lossy and it is necessary to employ an inline amplifier after the filtering process in order to bring signal levels up to soliton powers before further pulse compression using adiabatic amplification. Figure 15(a) shows a typical experimental configuration of an all-fiber integrated configuration, with a tunable diode laser output modulated by an inline lithium niobate frequency modulator, which is followed by an edge filter and power amplifier before launch into a long length of dispersion shifted fiber, which is counter directionally pumped by a high-power CW integrated fiber Raman laser enabling adiabatic Raman amplification and associated power-dependent compression of the input long pulses. On the right of Fig. 15, the power-dependent output pulse compression is shown, varying from about 8 ps to $290 \mathrm{fs}$ at $10 \mathrm{GHz}$ with an average power of $1.5 \mathrm{~W}$ at a pump power of $5 \mathrm{~W} .^{128}$

The above technique allows wavelength tunability and variable modulation rates over the range from 5 to $40 \mathrm{GHz}$; however, below about $5 \mathrm{GHz}$, the fundamental pulses require increased and impractical gain lengths for adiabatic compression. The technique can be applied throughout the mid-infrared; however, below $1270 \mathrm{~nm}$, it is not possible to obtain anomalous dispersion in single-mode silica-based optical fibers. Although photonic crystal fiber allows the dispersion zero to be selectively shifted toward the visible spectral region, once again impractical and expensive lengths of the fiber would need to be deployed and so negate their application; consequently, more 


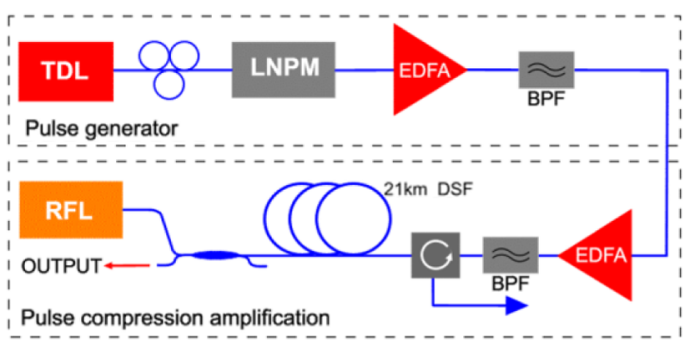

(a)

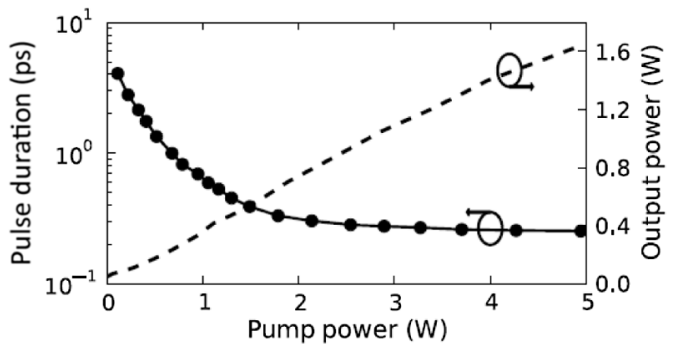

(b)

Fig. 15 (a) Experimental configuration of pulse source generated through spectral filtration of frequency modulated tuneable $\mathrm{CW}$ sources followed by sequential adiabatic compression through distributed Raman gain. (b) The variation of output pulse width and power with fundamental pump power is shown.

conventional dispersion compensation techniques, such as grating pair compressors, are used, with an associated loss of the all-fiber format. ${ }^{129}$ By avoiding sinusoidal or long pulse inputs and operating with seed pulses in the picosecond regime, tapered photonic crystal fibers have been demonstrated to enable adiabatic pulse compression to below $50 \mathrm{fs} .{ }^{130}$ Once such durations are achieved, propagation also gives rise to self-Raman interaction or the soliton self-frequency shift and this effect can also be utilized to give substantial wavelength tunability of the output.

\section{Fiber-Based Parametric Wavelength Conversion}

Using rare earth-doped lasers (Yb, Er, and Tm) and Raman conversion, the complete spectral region from about $1 \mu \mathrm{m}$ to the long wavelength transmission edge of silica-based fiber around $2.3 \mu \mathrm{m}$ can be comprehensively covered with numerous, temporally versatile fiber laser-based sources. Below $1 \mu \mathrm{m}$, however, there are no efficient silica-based fiber lasers and spectral coverage of the visible and near infrared requires a nonlinear process pumped by the above mentioned systems. Two principal schemes are used, direct second harmonic generation and FWM or parametric generation of the fundamental pumps.

A brief introduction to FWM and parametric generation in optical fiber was given in Sec. 2.3. Over the past few years, the technique has received significant attention because of the ability to generate wavelengths beyond those directly achievable with conventional fiber lasers with realization both in conventional ${ }^{131}$ and photonic crystal fibers. ${ }^{132}$ As schematically illustrated in Fig. 4, the use of scalar degenerate FWM in fibers leads to the production of widely spaced sidebands from a pump in the normal dispersion regime, where the generated signal and idler wavelengths are determined by the dispersive properties of the waveguide. Because the minimum zero dispersion wavelength of conventional optical fiber is limited to $\sim 1270 \mathrm{~nm}$, efficient generation to the visible is inhibited. This can be visualized empirically, with reference to the phase matching curve of Fig. 4, where if possible, signal wavelengths below about $900 \mathrm{~nm}$ would be phase matched with idler wavelengths $>2000 \mathrm{~nm}$, where the transmission loss significantly increases. Using photonic crystal fibers, however, it is possible to manipulate the dispersion zero enabling highly efficient FWM in the red ${ }^{133-135}$ and mid-infrared ${ }^{136}$ pumped by readily available high-power laser sources.

Fiberoptic parametric oscillators have also been demonstrated $^{137,138}$ and all-fiber oscillator configurations have been reported. ${ }^{139} \mathrm{~A}$ distinct advantage in using oscillator arrangements is the substantially reduced threshold powers as compared to single-pass systems; however, cavity configurations also have the disadvantages of fixed repetition rate and restricted control and selection of the operational pulsewidths. Consequently, greater versatility of the nonlinear source is obtained using MOPFA configurations to pump the fiber optic parametric generator in a singlepass configuration that allows wavelength tunability, pulse width, and pulse repetition rate selection, ${ }^{140}$ despite the caveat of an increased pump power requirement. A typical experimental configuration comprises of a fiber-integrated MOPFA coupled to a PCF in a simple single-pass arrangement. In Ref. 140, the seed signal was provided by a fiber-pigtailed semiconductor chip centered around $1.06 \mu \mathrm{m}$ that was wavelength tunable using a stress controlled fiber Bragg grating. This seed laser was tunable from 1055 to $1075 \mathrm{~nm}$ with a spectral linewidth of $0.07 \mathrm{~nm}$. This seed signal was modulated using a 10-GHz Mach-Zehnder amplitude modulator, driven by an electrical pulse generator, with pulse durations selectable from $300 \mathrm{ps}$ to $2 \mathrm{~ns}$ and at repetition rates from 1 to $30 \mathrm{MHz}$. The optically transmitted pulses were amplified in a fiber-integrated, two stage Yb-based configuration with inline filters to minimize the amplified spontaneous emission component. Peak pulse powers of several kilowatts were obtained. Parametric generation took place in a following polarization maintaining photonic crystal fiber, $3 \mathrm{~m}$ in length, which was chosen to minimize the influence of competing nonlinear effects, such as Raman generation, on the parametric generation process. The optimum length depends on the deployed pump conditions, such as pulse durations used and the effect of walk-off between pump and signal. In addition, processes such as SPM can affect the efficiency of the conversion process; however, in the system described here, a fiber length longer than optimum was used so that an extended range of pump pulse durations could be employed yet still give useful parametric output signal powers. By tuning the pump wavelength, the system was controllably tunable over the range from 0.74 to $0.8 \mu \mathrm{m}$ and this is illustrated on the left hand panel of Fig. 16 . The output pulse durations were selectable between 0.2 and $1.5 \mathrm{~ns}$, as shown in Fig. 16(b). Shorter pulses can be achieved though using different pulse generation techniques for the seed signal, but also can involve dispersion correction in the amplifiers and optimization of the nonlinear fiber lengths. In the system described, conversion efficiencies of up to $15 \%$ were achieved for the antiStokes signals and average powers in excess of $800 \mathrm{~mW}$ were obtained at $775 \mathrm{~nm}$.

It is possible to extend the short wavelength coverage using $1 \mu \mathrm{m}$ pumping by optimizing the phase matching process through the control of the dispersive properties of the nonlinear photonic fiber parameters. In addition, shorter wavelength 

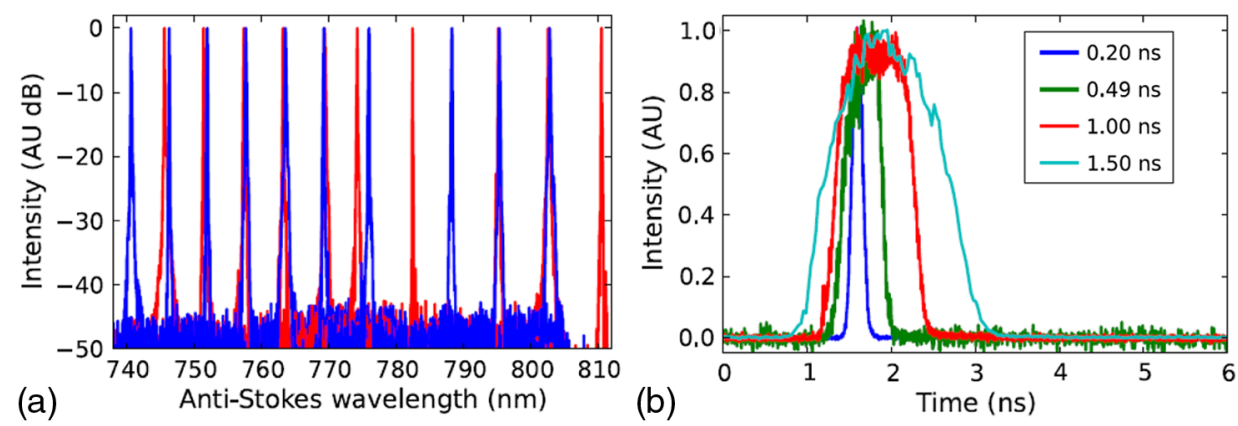

Fig. 16 (a) Wavelength tuning of the spectral output of a parametric signal with an average power exceeding $500 \mathrm{~mW}$ through wavelength tuning of the 300 ps pump pulses. (b) Temporal selectivity of the parametric signal through variation of the pump pulse duration.

pumps can be employed together with PCFs with short wavelength-shifted, dispersion zero wavelengths. ${ }^{141}$ As the dispersion zero shifts to shorter wavelengths, the inner core of the photonic crystal fibers decreases and coupling efficiencies generally tend to decrease. An additional problem is also that to maintain phase matching over the extended lengths of fiber used, exacting control of the fiber parameters is vital and places severe limits on feature sizes. Consequently, to generate visible wavelengths with average powers in the range of several watts, direct second harmonic generation of fiber laser outputs in bulk crystals or more commonly in periodically poled crystals is widely used.

\section{Visible Generation Through Second Harmonic Generation of Fiber Lasers}

The stimulated Raman process was briefly introduced in Sec. 2.5 and it described how efficient cascaded Stokes generation could be achieved and how simple Raman fiber lasers could be constructed using nested cavities in small core fiber cavities formed by sets of fiber Bragg gratings, with resonant wavelengths corresponding to the peak wavelength shifts of various cascaded Raman orders. ${ }^{33}$ In this way, the complete near infrared from an initial pump wavelength around $1030 \mathrm{~nm}$ supplied by a $\mathrm{Yb}$ fiber laser to beyond $2300 \mathrm{~nm}$ limited by silica fiber loss can be achieved. In addition to $\mathrm{CW}$ operation, pulsed outputs can be realized using synchronous pumping either from mode locked lasers or from high-power MOPFA schemes. When pumped such that the lasing is in the anomalously dispersive regime, soliton shaping can lead to femtosecond pulse generation ${ }^{142}$ or with Raman generation in the normally dispersive regime, dispersion compensation through the use of intracavity elements has also been shown to allow short pulse operation. ${ }^{143}$ By avoiding the pulse energy and power limitations of solitons and through operating with net normal dispersion, which has currently been termed as the dissipative soliton regime, substantially increased (several orders of magnitude) pulse energies are achieved, but with pulses that are highly chirped, which can be compressed to transform limited durations external to the resonator ${ }^{144}$ to enable femtosecond operation. In fiber Raman-based schemes, however, care should be taken with pump walk-off and cross-phase modulation giving nonlinear and hence noncompensatable chirp. Consequently, the synchronously pumped fiber Raman laser is capable of providing considerable temporal and wavelength versatility. These techniques are applicable to the visible, where Raman gain (which scales as $1 / \lambda$ ) is even higher; however, in germanium-doped silica-based fibers, high power pumping with visible radiation gives rise to photodarkening and associated transmission loss and cessation of laser action. Even with pure silica photonic crystal fibers, multiphoton ionization of high peak power pulsed visible inputs can be similarly problematic. For visible generation, therefore, second harmonic generation of versatile Raman-based sources in the near infrared by nonlinear crystals presents a more reliable resolution to these problems.

With the vast number of fluorophores being deployed in biophotonics, particularly in various fluorescence imaging techniques, excitation and de-excitation sources throughout the complete visible are required, with various temporal formats essential to optimize the particular technique deployed. Consequently, no single source can effectively meet all needs; however, some possible solutions exist with considerable parameter versatility. A good example of this is the source requirement for application of green fluorescent protein in stimulated emission depletion microscopy (STED). ${ }^{145}$ A pump wavelength around $560 \mathrm{~nm}$ is required, with a relatively high pulse energy, $>50 \mathrm{pJ}$ for good resolution, and an average power at a level such that detection is enabled without sample damage. The individual pulse duration should be in the range from 100 ps to $2 \mathrm{~ns}$ at a moderately low $\mathrm{MHz}$ repetition rate. In addition, a diffraction-limited beam quality is needed that is plane polarized and it is advantageous if the source is compact, efficient, and turn-key, requiring no operator intervention!

Naturally, there are many sources with highly competitive output characteristics that match some, but usually not all, of these requirements. Semiconductor diode laser systems offer compact and efficient formats, but generally exhibit poor beam quality and in conventional mode, locked cavities or gain switched formats provide pulse durations around 80 to $100 \mathrm{ps}$, with little selectivity, while average output powers are in the range of $1 \mathrm{~mW}$ at $\sim 80 \mathrm{MHz}$ giving pulse energies $\sim 7.5 \mathrm{pJ}$, that are not really sufficient for STED applications. Titanium-doped sapphire laser/amplifier pumped OPO schemes can meet the pulse energy requirements $(>10 \mathrm{~nJ})$ with average powers in the range of $1 \mathrm{~W}$ at a fixed $(\sim 80 \mathrm{MHz})$ repetition rate in pulses of $200 \mathrm{fs}$ also of fixed duration. Such schemes, although applicable, are costly, occupy a large footprint, and have a low overall efficiency. Spectral selection from a high average power supercontinuum does offer a practical solution in a compact footprint. Average powers of $\sim 10 \mathrm{~mW} / \mathrm{nm}$ can be obtained at $560 \mathrm{~nm}$ at typical fixed repetition rates around $80 \mathrm{MHz}$, consequently, nominally giving $\sim 2 \mathrm{~nJ}$ in a "pulse" with a $20-n m$ bandwidth. However, the typical optical efficiency with a $20-\mathrm{W}$ pump in this case is $\sim 1 \%$. 


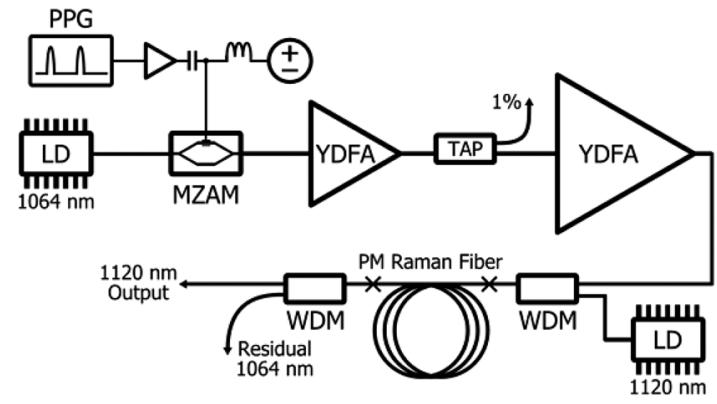

Fig. 17 Yb-MOPFA configuration used to synchronously pulse pump 1120-nm seeded single pass Raman generation in a short length polarization preserving fiber.

Synchronously pumped cascaded stimulated Raman generation in fiber with a fundamental wavelength provided by $\mathrm{Yb}$ MOPFA schemes, as described above and as shown in Fig. 17, affords a compact and efficient mechanism for wavelength selectivity throughout the spectral region from 1 to $2.3 \mu \mathrm{m}$, which can subsequently be frequency doubled to allow visible operation.

The technique of single-pass cascaded stimulated Raman generation in a single-mode optical fiber for production of wavelength diverse, subnanosecond sources synchronously pumped by a mode locked laser is well established and has been extensively used as a laboratory tool. ${ }^{44}$ Most commonly, the evolution of the Raman signal takes place from noise and as a consequence, extended lengths of fiber are required. As a result of these long interaction lengths, the effects of SPM and cross-phase modulation give rise to spectral broadening of the signals, while pump-signal walkoff can lead to complex phase structure on the generated pulses. In many cases, the spectral broadening is not problematic; however, where sequential frequency doubling is undertaken, particularly in long periodically poled nonlinear crystals for high conversion efficiency, the broadening can extend well beyond the phase matching bandwidths, with a consequential dramatic reduction in the efficiency attained. In synchronously pumped systems, with optimally short fiber gain lengths, seeding of the Raman signal, rather than allowing evolution from noise, significantly lowers the threshold powers and leads to output signal bandwidths that are primarily determined by the seed, ${ }^{146}$ thus allowing tunability as well as efficient conversion.

A representative experimental configuration is shown in Fig. 17. Although, as described above, various seed systems for the MOPFA can be utilized, in this example, duration tunable seed pulses were produced by modulating the output of a wavelength tunable $\mathrm{CW}$ pumped laser diode using a 10-GHz MachZehnder modulator with an extinction ration of better than $30 \mathrm{~dB}$. The optical output pulses from the biased modulator followed the electronic drive signals with a rise time of $\sim 60 \mathrm{ps}$, generating selectable seed pulses from 200 ps to 5 ns duration at selectable and variable repetition rates. Using two inline $\mathrm{Yb}$ doped fiber amplifiers, the seed pulses were amplified to average powers of $6 \mathrm{~W}$. These pump pulses at $1064 \mathrm{~nm}$ were combined with the output from a CW $1120 \mathrm{~nm}$ seed DFB diode with a maximum average power of $30 \mathrm{~mW}$, in a $2.2-\mathrm{m}$ long polarization preserving Raman gain fiber, with a gain coefficient of $\sim 2.5 \mathrm{~W}^{-1} \mathrm{~km}^{-1}$. The amplified, synchronously pumped Raman pulsed signal output was then directly focused into a 20-mm long periodically poled lithium tantalate crystal, with

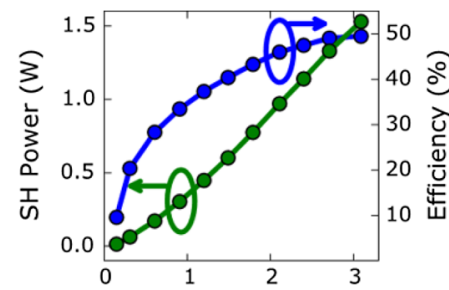

(a) Fundamental Power (W)

Fig. 18 (a) Variation of the frequency doubled synchronously pumped cascaded Raman signal at $560 \mathrm{~nm}$ with fundamental pump power at $1064 \mathrm{~nm}$ and (b) sampling oscilloscope traces of pulse width selection at $560 \mathrm{~nm}$ at different repetition rates.

a phase matching bandwidth of $0.15 \mathrm{~nm} .{ }^{147}$ It should also be noted that it is possible to fiber integrate the complete configuration by fiber pig-tailing the nonlinear crystal assembly. ${ }^{148}$ With up to $50 \%$ conversion efficiency obtained at $560 \mathrm{~nm}$ for pump powers of about $3 \mathrm{~W}$, as shown in Fig. 18, pulse durations were also selectable from 100 ps to 2.7 ns [see Fig. 18(b)] and repetition rates were variable from 2 to $50 \mathrm{MHz}$ in an effectively diffraction-limited beam. By tuning the wavelength of the seed laser and the phase matching temperature of the nonlinear crystal, wavelength selectivity ( $\sim 30$ to $40 \mathrm{~nm}$ ) throughout a single Raman gain band is possible. Extended wavelength coverage, however, is obtained through cascading the pump (and for longer wavelength operation using an $\mathrm{Yb}: \mathrm{Er}$ based pump) through various Raman orders and seeding with a narrow line laser at the desired wavelength of operation in the next order in the sequence, allowing broad tunability from 530 to $1000 \mathrm{~nm}$.

\section{Wavelength Tunable Mid-Infrared Generation via Difference Frequency Generation}

With increasing interest in diagnostics in the so-called "molecular fingerprint region," 2 to $12 \mu \mathrm{m}$, increased effort has been placed on the development of various sources covering this vitally important range. Since material and propagation losses in silica-based fibers inhibit most practical applications beyond about $2 \mu \mathrm{m}$, research has focused on the development of new fiber host materials that are transparent and/or nonlinear in the region. Heavily doped $\mathrm{GeO}_{2}$ fiber has allowed high-average power supercontinuum generation to beyond $3 \mu \mathrm{m} ;{ }^{149}$ however, to extend supercontinuum generation well into the molecular fingerprint region, fibers based upon fluorides ${ }^{150}$ or chalcogenides ${ }^{151}$ have enabled remarkable spectral coverage, up to $6.28 \mu \mathrm{m}$ with the former, while with the latter extended from 1.4 to $13.3 \mu \mathrm{m}$ albeit at an average power of $<1 \mathrm{~mW}$.

As has been discussed above, although many applications require wavelength diversity, these are usually met by restricted tuning ranges or specific target wavelength in more conventional laser formats and at higher operational power levels than conventionally supplied from supercontinuum sources. In the mid infrared, Cr- and Fe-doped ZnS- or ZnSe-based solid state lasers can provide average output powers in excess of $10-\mathrm{W}$ tunable in broad ranges of 2 to $3 \mu \mathrm{m}$ and $100 \mathrm{~s} \mathrm{~mW}$, from 3.7 to $5.00 \mu \mathrm{m}$, respectively, ${ }^{152}$ although the operation of the latter Fe-doped lasers was at cryogenic temperatures. Extended coverage throughout the molecular fingerprint region can alternatively be achieved using quantum cascade lasers ${ }^{153}$ and although operational powers at the watt level is achievable, cryogenic 
operation is often necessary and there is limited flexibility in the operational pulsewidths. Consequently, parametric conversion, ${ }^{154}$ which allows extensive tunability, diverse pulse formats, and high average powers depending on the selected combination of pump laser and nonlinear crystal, is becoming the practical source of choice in the 3 to $15 \mu \mathrm{m}$ range. As discussed in Sec. 6 , although Yb-fiber laser pumped optical parametric oscillators are capable of generating multiwatt average power tunable outputs in temporal formats ${ }^{155-157}$ from CW to femtosecond, the necessity of cavity configurations inhibits pulse repetition rates, restricts output pulsewidth variation and selectivity, while the resonators also require specialist broadband coatings and precision alignment. Single-pass optical parametric generation or difference frequency generation therefore simplifies the experimental configuration and as has been seen allows pulsewidth and repetition rate selectivity as well as broad tunability but usually at the expense of a raised threshold pump power for the generation process.

In the femtosecond regime, the single-pass difference frequency mixing of pulses from a fixed repetition rate, amplified mode-locked femtosecond Yb-fiber laser and its self-Raman (self-frequency) shifted signal allowed tunability from 3.0 to $4.4 \mu \mathrm{m}$ in a periodically poled lithium niobate (PPLN) crystal, ${ }^{158}$ while in the $\mathrm{CW}$ regime, the difference frequency mixing of $\mathrm{Yb}$ and Er fiber lasers at 1065 and $1550 \mathrm{~nm}$ in a 5-cm long PPLN crystal generated over $3.5 \mathrm{~W}$ of $\mathrm{CW}$ power at $3.4 \mu \mathrm{m} .{ }^{159}$ Extending this technique through the mixing of nanosecond pulses from a seeded, cascaded Yb-fiber amplifier chain at $1064 \mathrm{~nm}$ with a low power $(10 \mathrm{~mW})$ single frequency $\mathrm{CW}$ diode laser at $1525 \mathrm{~nm}$ resulted in greater than $1 \mathrm{~W}$ at $3520 \mathrm{~nm} .{ }^{160}$ By using a tunable diode seed, derived either from a narrow line diode laser or from a spectrally filtered ASE source together with picosecond pump pulses from a seeded Yb-MOPA, conversion efficiencies as high as $45 \%$ were reported over a quoted tuning range of 1450 to $3615 \mathrm{~nm}$ and average output powers of up to $3.5 \mathrm{~W} .^{161}$

The versatility of the seeded MOPFA in single-pass nonlinear generation was outlined above (see Secs. 6 and 7) and the technique has recently been expanded though the difference frequency generation of two wavelength tunable, pulsed (variable pulse width and repetition rate) seeded synchronized $\mathrm{Yb}$ - and Er-based systems in PPLN. ${ }^{162}$ The advantage of a common clock driving the pulsed seeding of the separate amplifiers means that the peak intensity requirement of the pump signal is reduced substantially below the level of the damage threshold of the nonlinear crystal used, whereas when CW seeding of the signal is deployed, there is a corresponding increased pump power requirement for efficient conversion. In addition, pulse synchronization is simplified with the inclusion of the common clock and variation of the output nonlinear pulse duration can also be finely tuned, simply by changing the temporal offset between the pump and signal at the nonlinear mixing crystal. The pulse duration can also be changed by simply changing the duration of the electronic drive signal to the laser systems seeding the MOPFAs, the wavelength of which can also be changed to permit broad tunability.

In the experiment, a Yb-based MOPFA output with 150-ps pulses and a linewidth of $0.13 \mathrm{~nm}$ at an average output power of $23 \mathrm{~W}$ was difference frequency mixed with the 400-ps signal pulses of linewidth $0.03 \mathrm{~nm}$ and an average power of $2.1 \mathrm{~W}$ around $1.559 \mathrm{~nm}$ in a $4 \mathrm{~cm}$ long PPLN temperature stabilized crystal, to give $3.5-\mathrm{W}$ average idler power at the difference

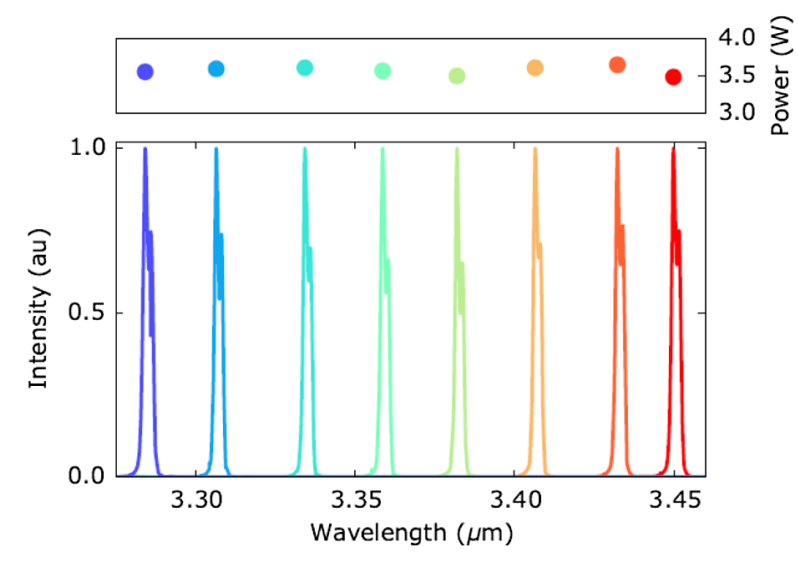

Fig. 19 Wavelength tunability through single pass difference frequency mixing in PPLN of a 150 ps pulsed Yb-MOPFA signal around $1063 \mathrm{~nm}$ with a $400 \mathrm{ps}$ tunable (1535 to $1570 \mathrm{~nm}$ ) signal from an ErMOPFA, with the corresponding idler average output power shown above.

frequency of $3.351 \mu \mathrm{m}$. By tuning of the signal wavelength from 1535 to $1570 \mathrm{~nm}$, tunability of the idler at was obtained, from 3.284 to $3.450 \mu \mathrm{m}$, while adjusting the temperature of the poled crystal for optimum phase matching. The output power was relatively constant, around $3.5 \mathrm{~W}$ across the generated spectral range. Figure 19 shows the tuning range and the associated average output power.

Extension of the tuning range should be possible through tuning of the pump signal. In addition, by utilizing seeded and cascaded fiber Raman MOPFA schemes, as described in Sec. 7, it should be noted that difference frequency mixing of the fundamental at $1.064 \mu \mathrm{m}$ with the cascaded second, third, and fourth Raman orders at 1.17, 1.24, and $1.31 \mu \mathrm{m}$ gives generation at $11,7.6$, and $5.7 \mu \mathrm{m}$, respectively, while tunable spectral seeding of the MOPFAs within each Raman gain band should allow broad tunability of the generated difference frequency, while retaining the temporal flexibility of repetition rate and pulse width of the seeded MOFFA configurations.

\section{Summary}

It is clear that no single laser source will ever match the diversity of requirements in the applications market. The purpose of this review was to illustrate the versatility in parameters available from compact, moderate power, fiber-based systems that could find biophotonic application. This, of course, is already the case for some of the devices. The supercontinuum source has been a remarkable scientific and commercial success providing unmatched wavelength coverage in a package with a compact footprint that effectively allows hands-free operation but has limited selectivity in pulse repetition rate, pulse duration and quality, as well as relatively low pulse energy. However, the supercontinnuum source has already been deployed in confocal reflectance microscopy, ${ }^{163}$ multiphoton fluorescence microscopy, ${ }^{164}$ conventional confocal fluorescence microscopy, ${ }^{165}$ and confocal microscopy coupled with fluorescence lifetime imaging. ${ }^{166}$ A near-infrared supercontinuum source in the spectral window around 1600 to $1870 \mathrm{~nm}$ has enabled deep penetration and minimal absorption allowing imaging of tissue with reduced scatter as a result of operation in long wavelengths deployed. ${ }^{167}$ To shorter wavelengths, a supercontinuum source has also been successfully deployed as the excitation source for STED. ${ }^{168}$ Spectral selection from a continuum, albeit 
a somewhat inefficient source of excitation radiation has also been used in CARS microscopy ${ }^{169}$ while the extensive spectral extent of the source has been attractive to application in optical coherence tomography. ${ }^{170}$ Many of the techniques listed here and more have been reviewed by Dunsby and French $^{52}$ in their article on the biophotonics applications of supercontinuum generation.

The MOPFA configuration on the other hand provides versatility in the parameters of pulse repetition rate, pulse duration, operational wavelength, and pulse energy with the added advantage of integrated nonlinear conversion as an add-on, either in fiber or a fiber-coupled nonlinear crystal package and is particularly applicable to operation in the visible and mid-infrared regions, where second harmonic generation of Raman-based schemes or difference frequency generation allows efficient spectral coverage. With the rapid uptake of numerous fluorophores and labels for application in PALM/STORM, STED, and RESOLFT, there is a need for excitation and switch-off wavelengths spanning the range from 400 to $750 \mathrm{~nm}$ and as was shown above, this can be provided by frequency doubled MOPFA pumped, seeded fiber Raman sources, while for multiphoton fluorescence excitation, femtosecond systems in the near infrared are required. The principal of operation of some of these sources has been described; however, it should be noted that there are many other widely applicable sources that have not been covered by this short review, for example, direct generation using semiconductor laser sources that can also be operated in MOPA configurations. It is, however, hoped that the versatility, simplicity, and efficiency of fiber-based schemes have been recognized, which may stimulate and lead to their broad application in biophotonics.

\section{Acknowledgments}

I wish to thank the members of the Femtosecond Optics Group at Imperial College for their input to results reported in this manuscript, in particular Dr. Robbie Murray, Dr. Tim Runcorn, Dr. Rob Woodward, Dr. Ed Kelleher and Dr. Sergei Popov. I acknowledge also the financial support of IPG Photonics Inc. and in particular Professor Valentin Gapontsev for his continued support. The EPSRC and the Royal Society also contributed to support of parts of the work reported herein.

\section{References}

1. V. Fomin et al., "10 kW single mode fiber laser," in Paper 1.3. 5th Symp. on High-Power Fiber Lasers, 14th Int. Conf. on Laser Optics, St. Petersburg, Russia (2010).

2. W. Liu et al., "Pre-chirp managed nonlinear amplification in fibers delivering 100 W, 60 fs pulses," Opt. Lett. 40, 151 (2015).

3. S. V. Chernikov et al., "1083 nm ytterbium doped fibre amplifier for optical pumping of helium," Electron. Lett. 33, 787 (1997).

4. E. M. Dianov, "Amplification in extended transmission bands using Bismuth-doped optical fibers," IEEE J. Lightwave Technol. 31, 681688 (2013).

5. P. A. Franken et al., "Generation of optical harmonics," Phys. Rev. Lett. 7, 118 (1961).

6. J. C. Knight et al., "All-silica single-mode optical fiber with photonic crystal cladding," Opt. Lett. 21, 1547 (1996).

7. P. St. J. Russell, "Photonic-crystal fibers," J. Lightwave Technol. 24, 4729-4749 (2006).

8. T. A. Birks, J. C. Knight, and P. St. J. Russell, "Endlessly single-mode photonic crystal fiber," Opt. Lett. 22, 961 (1997).

9. N. G. R. Broderick et al., "Nonlinearity in holey optical fibers: measurement and future opportunities," Opt. Lett. 24, 1395 (1999).
10. F. Shimizu, "Frequency broadening in liquids by a short light pulse," Phys. Rev. Lett. 19, 1097 (1967).

11. E. P. Ippen, C. V. Shank, and T. K. Gustavson, "Self-phase modulation of picosecond pulses in optical fibers," App. Phys. Lett. 24, 190 (1974).

12. E. B. Treacy, "Compression of picosecond light pulses," Phys. Lett. A 28, 34-35 (1968).

13. W. J. Tomlinson, R. H. Stolen, and C. V. Shank, "Compression of optical pulses chirped by self-phase modulation in fibers," J. Opt. Soc. Am. B 1, 139 (1984).

14. A. Hasegawa and F. Tappert, "Transmission of stationary nonlinear optical pulses in dispersive dielectric fibers," App. Phys. Lett. 23, 142 (1973).

15. L. F. Mollenauer, R. G. Stolen, and J. P. Gordon, "Experimental observation of picosecond pulse narrowing and solitons in optical fibers," Phys. Rev. Lett. 45, 1095 (1980).

16. L. F. Mollenauer and J. P. Gordon, Solitons in Optical Fibers Fundamentals and Applications, Elsevier Academic Press, Burlington, Massachusetts (2006).

17. C. J. S. de Matos et al., "All-fiber chirped pulse amplification using highly-dispersive air-core photonic bandgap fiber," Opt. Exp. 11, 2832 (2003).

18. J. Limpert et al., "All fiber chirped-pulse amplification system based on compression in air-guiding photonic bandgap fiber," Opt. Exp. 11, 3332 (2003).

19. J. Satsuma and N. Tajima, "B. Initial value problems of one-dimensional self-modulation of nonlinear waves in dispersive media," Prog. Theor. Phys. Suppl. 55, 284-306 (1974).

20. K. Tai and A. Tomita, "50x optical fiber pulse compression at $1.319 \mu \mathrm{m}$, , App. Phys. Lett. 56, 135 (1986).

21. A. B. Grudinin et al., "Stimulated-Raman scattering of $18 \mathrm{fs}$ pulses in $1.6 \mu \mathrm{m}$ region during pumping of a single mode optical fibre by the beam from a Nd:YAG laser $(\lambda=1.06 \mu \mathrm{m}), " J$. Exp. Theor. Phys. Lett. 45, 260 (1987).

22. A. S. Gouveia-Neto, A. S. L. Gomes, and J. R. Taylor, "Pulses of four optical cycles from an optimized optical/fibre grating pair/soliton pulse compressor," J. Mod. Opt. 35, 7-10 (1988).

23. R. H. Stolen, J. E. Bjorkholm, and A. Ashkin, "Phase-matched threewave mixing in silica fiber optical waveguides," App. Phys. Lett. 24, 308 (1974).

24. A. Hasegawa and F. Brinkman, "Tunable coherent IR and FIR sources utilizing modulational instability," IEEE J. Quantum Electron. 16, 694697 (1980).

25. K. Tai, A. Hasegawa, and A. Tomita, "Observation of modulational instability in optical fibers," Phys. Rev. Lett. 56, 135-138 (1986).

26. H. Itoh, G. M. Davis, and S. Sudo, "Continuous-wave pumped modulational instability in an optical fiber," Opt. Lett. 14, 1368 (1989).

27. A. S. Gouveia-Neto, M. E. Faldon, and J. R. Taylor, "Raman amplification of modulational instability and solitary wave formation," Opt. Lett. 13, 1029 (1988).

28. A. S. Gouveia-Neto et al., "Sub-picosecond pulse generation through cross phase modulation in optical fibers," Opt. Lett. 13, 901 (1988).

29. E. J. Greer et al., "Generation of $2 \mathrm{THz}$ repetition rate pulse trains through induced modulational instability," Electron. Lett. 25, 1246 (1989).

30. M. Nakazawa, K. Suzuki, and H. A. Haus, "The modulational instability laser-part 1: Experimental," IEEE J. Quantum Electron. 25, 2036-2044 (1989).

31. E. Yoshida and M. Nakazawa, "Low-threshold $115 \mathrm{GHz}$ continuous-wave mode-locked Er-doped fiber laser," Opt. Lett. 22, 1409 (1997).

32. R. H. Stolen, E. P. Ippen, and A. R. Tynes, "Raman oscillation in glass optical waveguides," Appl. Phys. Lett. 20, 62 (1972).

33. V. R. Supradeepa et al., "A highly efficient architecture for cascaded fiber Raman lasers," Opt. Exp. 21, 7148 (2013).

34. R. H. Stolen and E. P. Ippen, "Raman gain in glass optical waveguides," Appl. Phys. Lett. 22, 276 (1973).

35. C. Lin, V. T. Nguyen, and W. G. French, "Wideband near I.R. continuum $(0.7-2.1 \mu \mathrm{m})$ generated in low loss optical fibres," Electron. Lett. 14, 822 (1978).

36. E. M. Dianov et al., "Stimulated-Raman conversion of multisoliton pulses in quartz optical fibers," J. Exp. Theor. Phys. Lett. 41, 294 (1985). 
37. F. M. Mitschke and L. F. Mollenauer, "Discovery of the soliton selffrequency shift," Opt. Lett. 11, 659-661 (1986).

38. J. P. Gordon, "Theory of the soliton self-frequency shift," Opt. Lett. 11, 662 (1986).

39. R. R. Alfano and S. L. Shapiro, "Observation of self-phase modulation and small-scale filaments in crystals and glasses," Phys. Rev. Lett. 24, 592 (1970).

40. R. R. Alfano and S. L. Shapiro, "Emission in the region 4000 to 7000 Å via four photon coupling in glass," Phys. Rev. Lett. 24, 584 (1970).

41. R. R. Alfano and S. L. Shapiro, "Picosecond spectroscopy using the inverse Raman effect," Chem. Phys. Lett. 8, 631-633 (1971).

42. R. L. Fork et al., "Femtosecond white light continuum pulses," Opt. Lett. 8, 1 (1983).

43. C. Lin and R. H. Stolen, "New nanosecond continuum for excited state spectroscopy," Appl. Phys. Lett. 28, 216 (1976).

44. L. G. Cohen and C. Lin, "A universal fiber optic (UFO) measurement system based upon a near-IR fiber Raman laser," IEEE J. Quantum Electron. 14, 855-859 (1978).

45. A. S. Gouveia-Neto, A. S. L. Gomes, and J. R. Taylor, "High efficiency single-pass soliton-like compression of Raman radiation in an optical fiber around $1.4 \mu \mathrm{m}, "$ Opt. Lett. 12, 1035 (1987).

46. P. Beaud et al., "Ultrashort pulse propagation, pulse breakup and fundamental soliton formation in a single mode optical fiber," IEEE $J$. Quantum Electron. 23, 1938-1946 (1987).

47. J. K. Ranka, R. S. Windeler, and A. J. Stentz, "Visible supercontinuum generation in air-silica microstructure optical fibers with anomalous dispersion at $800 \mathrm{~nm}$, , Opt. Lett. 25, 25 (2000).

48. A. B. Rulkov et al., "High brightness picosecond all-fiber generation in 525-1800 nm range with picosecond Yb pumping," Opt. Exp. 13, 377 (2005).

49. K. K. Chen et al., "Picosecond fiber MOPFA pumped supercontinuum source with 39 W output power," Opt. Exp. 18, 5426 (2010).

50. X. Hu et al., "High average power, strictly all-fiber supercontinuum source with good beam quality," Opt. Lett. 36, 2659 (2011).

51. J. C Travers et al., "Visible supercontinuum generation in photonic crystal fibre with a 400 W CW fibre laser," Opt. Exp. 16, 14435 (2008).

52. C. Dunsby and P. M. W. French, "Biophotonics applications of supercontinuum generation," Chapter 15 in Supercontinuum Generation in Optical Fibers, J. M. Dudley and J. R. Taylor, Eds., Cambridge University Press, New York (2012).

53. E. M. Dianov et al., "Optimal compression of multi-soliton pulses in optical fibers," Sov. Tech. Phys. Lett. 12, 311 (1986).

54. J. Hermann et al., "Experimental evidence for supercontinuum generation by fission of high-order solitons in photonic crystal fibers," Phys. Rev. Lett. 88, 173901 (2002).

55. J. M. Dudley, G. Genty, and S. Coen, "Supercontinuum generation in photonic crystal fiber," Rev. Mod. Phys. 78, 1135 (2006).

56. G. Genty, S. Coen, and J. M. Dudley, "Fiber-supercontinuum sources," J. Opt. Soc. Am. B 24, 1771-1785 (2007).

57. J. M. Dudley et al., "Cross-correlation frequency resolved optical gating analysis of broadband continuum generation in photonic crystal fiber: simulations and experiment," Opt. Exp. 10, 1215 (2002).

58. D. R. Solli et al., "Optical rogue waves," Nature 450, 1054-1057 (2007).

59. J.M. Dudley, G. Genty, and B. Eggleton, "Harnessing and control of rogue waves in supercontinuum generation," Opt. Exp. 16, 3644 (2008).

60. G. A. Nowak, J. Kim, and M. N. Islam, "Stable supercontinuum generation in short lengths of conventional dispersion shifted fiber," App. Opt. 38, 7364-7369 (1999).

61. A. M. Heidt, "Pulse preserving flat-top supercontinuum generation in all-normal dispersion photonic crystal fiber," J. Opt. Soc. Am. B 27 , 550-559 (2010)

62. B. A. Cumberland et al., " $29 \mathrm{~W}$ High power $\mathrm{CW}$ supercontinuum source," Opt. Exp. 16, 5954 (2008).

63. H. W. Mocker and R. J. Collins, "Mode competition and self-locking effects in a Q-switched ruby laser," App. Phys. Lett. 7, 270 (1965).

64. M. I. Dzhibladze et al., "Mode locking in a fiber laser," Sov. J. Quantum Electron. 13, 245 (1983).

65. P. M. W. French, P. G. J. Wigley, and J. R. Taylor, "Mode-locking of a continuous wave neodymium doped fibre laser with a linear external cavity," Electron. Lett. 26, 1238 (1990).
66. N. J. Doran and D. Wood, “Nonlinear optical loop mirror," Opt. Lett. 13, 56 (1988).

67. M. E. Fermann et al., "Nonlinear amplifying loop mirror," Opt. Lett. 15 , 752 (1990).

68. R. H. Stolen, J. Botineau, and A. Ashkin, "Intensity discrimination of optical pulses with birefringent fibers," Opt. Lett. 7, 512 (1982).

69. M. Hofer et al., "Mode locking with cross-phase and self-phase modulation," Opt. Lett. 16, 502 (1991).

70. V. J. Matsas et al., "Self-starting passively mode-locked fibre ring soliton laser exploiting nonlinear polarization rotation," Electron. Lett. 28, 1391 (1992)

71. V. J. Matas, T. P. Newson, and M. N. Zervas, "Self-starting passively mode locked fibre ring laser exploiting non-linear polarization switching," Opt. Commun. 92, 61-66 (1992).

72. I. N. Duling, III, "Sub-picosecond all-fibre erbium laser," Electron. Lett. 27, 544 (1991).

73. D. A. Chestnut and J. R. Taylor, "Wavelength-versatile subpicosecond pulsed lasers using Raman gain in figure-of-eight fiber geometries," Opt. Lett. 30, 2982 (2005).

74. C. Aguergaray et al., "Mode-locked femtosecond all-normal all-PM Ybdoped fiber laser using a nonlinear amplifying loop mirror," Opt. Exp. 20, 10545 (2012)

75. M. Zirngibl et al., " 1.2 ps pulses from passively mode-locked laser diode pumped Er-doped fibre ring laser," Electron. Lett. 27, 1734 (1991).

76. E. A. deSouza et al., "Saturable absorber mode-locked polarization maintaining erbium-doped fibre laser," Electron. Lett. 29, 447 (1993).

77. M. Rusu et al., "Femtosecond neodymium-doped fiber laser operating in the 894-909-nm spectral range," IEEE Photonics Technol. Lett. 16, 1029-1031 (2004)

78. O. G. Okhotnikov et al., "Mode-locked ytterbium fiber laser tunable in the 980-1070-nm spectral range," Opt. Lett. 28, 1522 (2003).

79. R. Gumenyuk et al., " $1.32 \mu$ m mode-locked Bismuth-doped fiber laser operating in anomalous and normal dispersion regimes," Opt. Lett. 38, 4005 (2013)

80. O. G. Okhotnikov, A. B. Grudinin, and M. Pessa, "Ultra-fast fibre laser systems based on SESAM technology: new horizons and applications," New J. Phys. 6, 177 (2004).

81. M. A. Chernysheva et al., "SESAM and SWCNT mode-locked all-fiber Thulium-doped lasers based on the nonlinear amplifying loop mirror," IEEE J. Sel. Top. Quantum Electron. 20, 1101208 (2104).

82. A. Chamorovskiy et al., "Raman fiber laser pumped by a semiconductor disk laser and mode locked by a semiconductor saturable absorber mirror," Opt. Lett. 35, 3529 (2010).

83. S. Y. Set et al., "Laser mode locking using a saturable absorber incorporating carbon nanotubes," J. Lightwave Technol. 22, 51-56 (2004).

84. S. Kivistö et al., "Carbon nanotube films for ultrafast broadband technology," Opt. Exp. 17, 2358 (2009).

85. J. C. Travers et al., "Using the $\mathrm{E}_{22}$ transition of carbon nanotubes for fiber laser mode-locking," Laser Phys. Lett. 8, 144 (2011).

86. A. Hasegawa and Y. Kodama, "Guiding center soliton," Phys. Rev. Lett. 66, 161 (1991).

87. S. M. J. Kelly et al., "Average soliton dynamics of a high- gain erbium fiber laser," Opt. Lett. 16, 1337 (1991).

88. S. M. J. Kelly, "Characteristic sideband instability of periodically amplified average soliton," Electron. Lett. 28, 806 (1992).

89. N. Pandit et al., "Characteristic instability of fibre loop soliton lasers," Electron. Lett. 28, 455 (1992).

90. N. J. Smith, K. J. Blow, and I. Andonovic, "Sideband generation through perturbations to the average soliton model," J. Lightwave Technol. 10, 1329-1333 (1992).

91. S. Kobtsev, S. Kukarin, and Y. Fedotov, "Ultra-low repetition rate mode-locked fiber laser with high-energy pulses," Opt. Exp. 16, 21936 (2008)

92. R. I. Woodward et al., "Fiber grating compression of giant-chirped nanosecond pulses from an ultra-long nanotibe mode-locked fiber laser," Opt. Lett. 40, 387 (2015).

93. M. Zhang et al., "Nanosecond pulse generation in lumped normally dispersive all-fiber mode-locked laser," Photonics Technol. Lett. 23, 1379 1381 (2011)

94. E. J. R. Kelleher et al., "Bismuth fiber integrated laser mode-locked by carbon nanotubes," Laser Phys. Lett. 7, 790 (2010). 
95. Q. Bao et al., "Atomic-layer graphene as a saturable absorber for ultrafast pulsed lasers," Adv. Funct. Mater. 19, 3077-3083 (2009).

96. L. M. Zhao et al., "Dissipative soliton operation of an ytterbium-doped fiber laser mode locked with atomic multilayer graphene," Opt. Lett. 35, 3622 (2010).

97. M. Zhang et al., "Tm-doped fiber laser mode-locked by graphene-polymer composite," Opt. Exp. 20, 25077 (2012).

98. Z. Sun, T. Hasan, and A.C. Ferrari, "Ultrafast lasers mode-locked by nanotubes and graphene," Phys. E 44, 1082-1091 (2012).

99. S. G. Grubb et al., " $1.3 \mu \mathrm{m}$ cascaded Raman amplifier in germanosilicate fibers," in Paper PD3 Proc. of Opt. Amp. and their Applications, Breckenridge, Colorado (1994).

100. C. E. S. Castellani et al., "Ultrafast Raman laser mode-locked by nanotubes," Opt. Lett. 36, 3996 (2011).

101. C. E. S. Castellani et al., "Harmonic and single pulse operation of a Raman laser using graphene," Laser. Phys. Lett. 9, 223 (2012).

102. C. E. S. Castellani et al., "CW-pumped short pulsed $1.12 \mu \mathrm{m}$ Raman laser using carbon nanotubes," Laser Phys. Lett. 10, 015101 (2013).

103. K. Wang et al., "Ultrafast saturable absorption of two-dimensional $\mathrm{MoS}_{2}$ nanosheets," ACS Nano 7, 9260-9267 (2013).

104. H. Zhang et al., "Molybdenum disulfide $\left(\mathrm{MoS}_{2}\right)$ as a broadband saturable absorber for ultra-fast photonics," Opt. Exp. 22, 7249 (2014).

105. Z. Tian et al., "Mode-locked thulium fiber laser with $\mathrm{MoS}_{2}$," Laser Phys. Lett. 12, 065104 (2015).

106. M. Zhang et al., "Solution processed $\mathrm{MoS}_{2}-\mathrm{PVA}$ composite for subbandgap mode-locking of a wideband tunable ultrafast Er:fiber laser," Nano Res. 8, 1522-1534 (2015).

107. R. Khazaeizhad et al., "Mode-locking of Er-doped fiber laser using a multilayer $\mathrm{MoS}_{2}$ thin film as a saturable absorber in both anomalous and normal dispersion regimes," Opt. Exp. 22, 23732 (2014).

108. D. Mao et al., "WS ${ }_{2}$ mode-locked ultrafast fiber laser," Sci. Rep. 5, 7965 (2015).

109. M. Jung et al., "Mode-locked, $1.94-\mu \mathrm{m}$, all-fiberized laser using $\mathrm{WS}_{2}$-based evanescent field interaction," Opt. Exp. 23, 19996 (2015).

110. Z. Luo et al., "Nonlinear optical absorption of few-layer molybdenum diselenide $\left(\mathrm{MoSe}_{2}\right)$ for passively mode-locked soliton fiber laser," Photonics Res. 3, A79 (2015).

111. J. Sotor et al., "Black phosphorus saturable absorber for ultrashort pulse generation," App. Phys. Lett. 107, 051108 (2015).

112. J. Lee et al., "Femtosecond pulse erbium fiber laser incorporating a saturable absorber based on bulk-structured $\mathrm{Bi}_{2} \mathrm{Te}_{3}$ topological insulator," Opt. Exp. 22, 6165 (2014).

113. H. Liu et al., "Femtosecond pulse generation from a topological insulator mode-locked fiber laser," Opt. Exp. 22, 6868 (2014).

114. J. Sotor et al., "Mode-locked erbium-doped fiber laser based on evanescent field interaction with $\mathrm{Sb}_{2} \mathrm{Te}_{3}$ topological insulator," App. Phys. Lett. 104, 251112 (2014).

115. M. Zhang et al., "Characterization of nonlinear saturation and modelocking potential of ionically-doped colored glass filter for short-pulse fiber lasers," Opt. Exp. 21, 12562 (2013).

116. A. Hasegawa and Y. Kodama, "Signal transmission by optical solitons in monomode fiber," Proc IEEE 69, 1145-1150 (1981).

117. K. Tajima, "Compensation of soliton broadening in nonlinear optical fibre with loss," Opt. Lett. 12, 54 (1987).

118. N. J. Zabusky and M. D. Kruskal, "Interaction of solitons in a collisionless plasma and the recurrence of initial states," Phys. Rev. Lett. 15, 240-243 (1965).

119. E. M. Dianov et al., "Generation of a train of fundamental solitons at a high repetition rate in optical fibers," Opt. Lett. 14, 1008 (1989).

120. P. V. Mamyshev, S. V. Chernikov, and E. M. Dianov, "Generation of fundamental soliton trains for high-bit-rate optical fiber communication lines," IEEE J. Quantum Electron. 27, 2347-2355 (1991).

121. S. V. Chernikov et al., "Generation of soliton pulse train in optical fibre using two CW single mode diode lasers," Electron. Lett. 28, 931-932 (1992).

122. S. V. Chernikov, J. R. Taylor, and R. Kashyap, "Comblike dispersionprofiled fiber for soliton pulse train generation," Opt. Lett. 19, 539 (1994).

123. S. V. Chernikov, J. R. Taylor, and R. Kashyap, "Experimental demonstration of step-like dispersion profiling in optical fibre for soliton pulse generation and compression," Electron. Lett. 30, 433-435 (1994).

124. P. C. Reeves-Hall and J. R. Taylor, "Wavelength and duration tunable sub-picosecond source using adiabatic Raman compression," Electron. Lett. 37, 417 (2001).

125. C. J. S. de Matos, D. A. Chestnut, and J. R. Taylor, "Wavelength- and duration-tunable soliton source based on a $20 \mathrm{GHz}$ Mach-Zhender modulator and adiabatic Raman compression," Appl. Phys. Lett. 81, 2932 (2002).

126. A. S. L. Gomes et al., "Optical pulse narrowing by the spectral windowing of self phase modulated picosecond pulses," Opt. Соттип. 59, 399-404 (1986).

127. P. V. Mamyshev, "Dual-wavelength source of high repetition rate transform limited optical pulses for soliton transmission," Opt. Lett. 19, 2074 (1994).

128. B. H. Chapman et al., "All-fiber integrated $10 \mathrm{GHz}$ repetition rate femtosecond laser source based on Raman compression of pulses generated through spectral masking of a phase-modulated diode," Opt. Lett. 37, 3099 (2012).

129. B. H. Chapman et al., "Femtosecond pulses at $20 \mathrm{GHz}$ repetition rate through spectral masking of a phase modulated signal and nonlinear pulse compression," Opt. Exp. 21, 5671-5676 (2013).

130. J. C. Travers et al., "Optical pulse compression in dispersion decreasing photonic crystal fibers," Opt. Exp. 15, 13203 (2007).

131. G. K. L. Wong et al., "High-conversion-efficiency widely-tunable allfiber optical parametric oscillator," Opt. Exp. 15, 2947 (2007).

132. J. D. Harvey et al., "Scalar modulation instability in the normal dispersion regime by use of a photonic crystal fiber," Opt. Lett. 28, 2225 (2003).

133. D. Nodop et al., "Efficient high-power generation of visible and midinfrared light by degenerate four-wave-mixing in a large-mode-area photonic-crystal fiber," Opt. Lett. 34, 3499 (2009).

134. L. Lavoute et al., "High power red and near-IR generation using four wave mixing in all integrated fibre laser systems," Opt. Exp. 18, 16193 (2010).

135. C. Jauregui et al., "High-power efficient generation of visible and midinfrared radiation exploiting four-wave mixing in optical fibers," Opt. Exp. 20, 24957 (2012).

136. A. Herzog, A. Shamir, and A. A. Ishaaya, "Wavelength conversion of nanosecond pulses to the mid-IR in photonic crystal fibers," Opt. Lett. 37, 82 (2012).

137. J. E. Sharping, "Microstructure fiber based optical parametric oscillators," J. Lightwave Technol. 26, 2184-2191 (2008).

138. R. T. Murray et al., "Synchronously pumped photonic crystal fiber-based optical parametric oscillator," Opt. Lett. 37, 3156 (2012).

139. G. Van der Westhuizen and J. Nilsson, "Fiber optical parametric oscillator for large frequency-shift wavelength conversion," IEEE J. Quantum Electron. 47, 1396-1403 (2011).

140. R. T. Murray et al., "Widely tunable polarization maintaining photonic crystal fiber based parametric wavelength conversion," Opt. Exp. 21, 15826 (2013).

141. D. J. J. Hu et al., "Fiber-integrated $780 \mathrm{~nm}$ source for visible parametric generation," Opt. Exp. 22, 29726 (2014).

142. A. S. Gouveia-Neto, A. S. L. Gomes, and J. R. Taylor, "A femtosecond soliton Raman ring laser," Electron. Lett. 23, 537-538 (1987).

143. J. D. Kafka, D. F. Head, and T. Baer, "Dispersion compensated fiber Raman oscillator," in Ultrafast Phenomena V, Springer Series in Chemical Physics, Vol. 46, p. 51, Springer, Berlin (1986).

144. D. Churin et al., "High-power synchronously pumped femtosecond Raman fiber laser," Opt. Lett. 40, 2529 (2015).

145. S. W. Hell and J. Wichmann, "Breaking the diffraction resolution limit by stimulated emission: stimulated-emission-depletion fluorescence microscopy," Opt. Lett. 19, 780 (1994).

146. E. E. Rowen et al., "A combined Yb-Raman fiber amplifier for generating narrow linewidth, high-power pulses in the $1100 \mathrm{~nm}-1200 \mathrm{~nm}$ wavelength range and efficient nonlinear conversion to yellow," Proc. SPIE 8601, 86011J (2013).

147. T. H. Runcorn et al., "Duration-tunable picosecond source at $560 \mathrm{~nm}$ with watt level average power," Opt. Lett. 40, 3085 (2015).

148. T. H. Runcorn et al., "Fiber-integrated frequency-doubling of a picosecond Raman laser to 560 nm," Opt. Exp. 23, 15728 (2015). 
149. M. Zhang et al., "Mid-infrared Raman-soliton continuum pumped by a nanotube-mode-locked sub-picosecond Tm-doped MOPFA," Opt. Exp. 21, 23261 (2013).

150. G. Qin et al., "Ultrabroadbandsupercontinuum generation from ultraviolet to $6.28 \mu \mathrm{m}$ in a fluoride fiber," Appl. Phys. Lett. 95, 161103 (2009).

151. C. R. Petersen et al., "Mid-infrared supercontinuum covering the $1.4-$ $13.3 \mu \mathrm{m}$ molecular fingerprint region using ultra-high NA chalcogenide step-index fibre," Nat. Photonics 8, 830-834 (2014).

152. S. B. Mirov et al., "Progress in Mid-IR lasers based on $\mathrm{Cr}$ and FeDoped II-VI chalcogenides," IEEE J. Sel. Top. Quantum Electron. 21, 292-310 (2015).

153. Y. Yao, A. J. Hoffman, and C. F. Gmachl, "Mid-infrared quantum cascade lasers," Nat. Photonics 6, 432-439 (2012).

154. V. Petrov, "Progress in $1 \mu \mathrm{m}$-pumped mid IR optical parametric oscillators based on non-oxide nonlinear crystals," IEEE J. Sel. Top. Quantum Electron. 21, 193 (2015).

155. F. Adler et al., "Phase-stabilized, $1.5 \mathrm{~W}$ frequency comb at $2.8-4.8 \mu \mathrm{m}, "$ Opt. Lett. 34, 1330 (2009).

156. L. Xu et al., "Fiber-laser-pumped, high-energy, mid-IR, picosecond optical parametric oscillator with a high-harmonic cavity," Opt. Lett. 40, 3288 (2015).

157. M. Ebrahim-Zadeh and S. Chaitanya Kumar, "Yb-fiber-laser-pumped ultrafast frequency conversion sources from the mid-infrared to the ultraviolet," IEEE J. Sel. Top. Quantum Electron. 20, 624 (2014).

158. T. W. Neely, T. A. Johnson, and S. A. Diddams, "High-power broadband laser source tunable from $3.0 \mu \mathrm{m}$ to $4.4 \mu \mathrm{m}$ based on a femtosecond Yb:fiber oscillator," Opt. Lett. 36, 4020 (2011).

159. S. Guha, J. O. Barnes, and L. P. Gonzalez, "Multiwatt-level continuous wave midwave infrared generation using difference frequency mixing in periodically poled $\mathrm{MgO}$-doped lithium niobate," Opt. Lett. 39, 5018 (2014).

160. P. Belden, D.W. Chen, and F. di Teodoro, "Watt-level, gigahertz-linewidth difference frequency generation in PPLN pumped by a nanosecond-pulse fiber laser source," Opt. Lett. 40, 958 (2015).

161. L. Xu et al., "High-energy, near-and mid-IR picosecond pulses generated by a fiber0-MOPA-pumped optical parametric generator and amplifier," Opt. Exp. 23, 12613 (2015).
162. R. T. Murray et al., "Highly efficient mid-infrared difference frequency generation using synchronously pulsed fiber lasers," Opt. Lett. 41, 2446-2449 (2016).

163. J. Garzon et al., "Chromatic confocal microscopy by means of continuum light generated through a standard single mode fibre," $J$. Opt. A: Pure App. Opt. 6, 544 (2004).

164. J. E. Jureller et al., "Widely tunable femtosecond pulses from a tapered fiber for ultrafast microscopy and multiphoton applications," in Ultrafast Phenomena XIII, Springer Verlag, Berlin (2003).

165. G. McConnell, "Confocal laser scanning fluorescence microscopy with a visible continuum source," Opt. Exp. 12, 2844 (2004).

166. C. Dunsby et al., "An electronically tunable ultrafast laser source applied to fluorescence imaging and fluorescence lifetime imaging microscopy," J. Phys. D-App. Phys. 37, 3296 (2004).

167. L. A. Sordillo et al., "Deep optical imaging of tissue using the second and third near-infrared spectral windows," J. Biomed. Opt. 19, 056004 (2014).

168. E. Auksorius et al., "Stimulated emission depletion microscopy with a supercontinuum source and fluorescence lifetime imaging," Opt. Lett. 33, 113 (2008).

169. H. N. Paulsen et al., "Coherent anti-Stokes Raman scattering microscopy with a photonic crystal fiber based light source," Opt. Lett. 28, 1123 (2003).

170. I. Hartl et al., "Ultrahigh-resolution optical coherence tomography using continuum generation in an air-silica microstructure optical fiber," Opt. Lett. 26, 608 (2001).

Roy Taylor received his PhD in 1974 from the Queen's University of Belfast. He established the Femtosecond Optics Group at Imperial College in 1986. His research interests have included tunable femtosecond dye lasers, amplifiers and nonlinear conversion, ultrafast molecular dynamics, ultrashort fibre laser sources, compact solid state lasers, optical soliton generation and nonlinear optics in fibres, publishing more than 390 articles in international peer-reviewed journals and coauthoring over 400 conference presentations in these areas. 